\title{
Die Motive Reden, Schweigen und Hören als Mittel brieflicher Kommunikation
}

\author{
Ihre Thematisierung in den Ignatianen
}

\begin{abstract}
Based on the assumption that the middle recension of the Ignatian Corpus is pseudepigraphic, written and designed probably ca. 170 in Rome or Asia Minor as an integral collection of seven letters, this chapter identifies and discusses the literary themes - spread across the collection - of "speaking", "being silent" and "listening" as motifs designed to help drive the rhetorical purpose of the letters, that is the author's exhortation to his addressees regarding what he considers to be the true doctrine ( $\gamma \nu \omega \dot{\mu \eta})$, the rejection of false teachings, the authority of the single غंлібколоৎ and the meaning of martyrdom. The chapter concludes with some observations regarding the reception of the letter corpus, beginning with Irenaeus adv. haer. 5.28.4.

Seit der Auffindung des Corpus Ignatianum im 15. Jahrhundert sind „Zweifel an Ursprünglichkeit und Echtheit der Briefe Signum der Ignatianen-Forschung“" , so Ferdinand Prostmeier, dem die folgenden Ausführungen gewidmet sein sollen, im Lemma „Ignatius von Antiochia“ in der dritten Auflage des Lexikons der antiken christlichen Literatur. Bezeugt wurden sieben Briefe des Ignatios, der Bischof von Antiochia ${ }^{2}$ gewesen und unter Kaiser Trajan das Martyrium erlitten haben soll, ${ }^{3}$ zwar bereits von Eusebius, u. a. in seiner Kirchengeschichte. ${ }^{4}$ Die erstmals 1498 zunächst unvollständig, 1536 vollständig gedruckte „längere Rezension“ enthielt jedoch mehr als sieben Briefe, ${ }^{5}$ von denen einige offensichtlich nicht aus dem 2. Jahrhundert
\end{abstract}

1 Prostmeier (2002) 347. Der Text der Ignatios-Briefe wird im folgenden nach Ehrman (2003) 201321 zitiert.

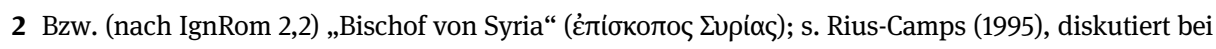
Prostmeier (2002) 347; Brent (2009) 100-109; Theobald (2016) 294.

3 Vgl. Eus., Hier. chron. an. 68 (GCS 24; Eusebius 7, 186.16-17): Antiochiae secundus episcopus ordinatur Ignatius; an. 108 (ebd. 194.2.24): Ignatius quoque antiochenae ecclesiae episcopus Romam perductus bestiis traditur; post quem tertius constitutur episcopus Heron; ausführlich zu den Angaben bei Eusebius, Lechner (1999) 75-115.

4 Eus., hist. eccl. 3,36,3-15. Nach Eusebius schrieb Ignatios, der sich in Gefangenschaft und auf dem Weg von Antiochia nach Rom zu seiner Hinrichtung im Amphitheater (ad bestias) befand, in Smyrna IgnEph, IgnMagn, IgnTrall und IgnRom, und danach in Troas IgnPhld, IgnSm und IgnPol; s. Prostmeier (2002) 346; Löhr (2009) $104 f$.

Prof. Dr. Josef Lössl, Cardiff University, School of History, Archaeology and Religion, Professor of Historical Theology and Intellectual History, e-mail: LosslJ@cardiff.ac.uk 
stammten. ${ }^{6}$ Als im 17. Jahrhundert weitere - kürzere - Rezensionen entdeckt wurden, ${ }^{7}$ die eher mit dem Zeugnis des Eusebius im Einklang schienen, setzte dies den Zweifeln keineswegs ein Ende, im Gegenteil. ${ }^{8}$ War die früheste Echtheitskritik (wie

5 Nämlich (je nach Zählung) 17, 13, 12 oder 11 Briefe. Allgemeines bei Schoedel (1990) 25; 21 Anm. 5 (Literatur); Löhr (2009) 105-6; siehe auch im vorliegenden Band das Kapitel von Hanns Christof Brennecke („Die recensio longior des Corpus Ignatianum“). Vier nur auf Latein erhaltene und wohl auch in Latein abgefasste Briefe stammen aus dem Mittelalter (12. Jh.?). Ein Brief, von Maria von Kassobola, ist an Ignatios adressiert. Diese fünf Briefe fehlen im Wiegendruck des Faber Stapulensis von 1498, einer Ausgabe der Werke des Dionysius Areopagita in einer lateinischen Übersetzung Ambrogio Traversaris, der neben dem Brief des Polykarp elf Briefe des Ignatios zählt. Die Übersetzung des Ignatios-Materials stammt nicht von Traversari. Der Titel dieser Ausgabe lautet: Dionysii Celestis hierarchia, Ecclesiastica hierarchia, Divina nomina, Mystica theologia, Undecim epistole. Ignatii Undecim epistole. Polycarpi Epistola una. Der Band schließt mit In alma Parhisiorum schola per Ioannem Higmanū et Wolfgangū Hopylium artis formularie socios. Anno ab incarnatione eiusdem domini nostri Ihesu Christi 1498 die sexto Februarii; s. Lagarde (1882) ii-iii; Die Kölner Ausgabe des Symphorianus Champerius von 1529 (bzw. 1536) enthält alle auf Latein erhaltenen Briefe; s. Amelungk (1899) 3-4 sowie 4 Anm. 1. Die erste Ausgabe eines griechischen Textes wurde 1557 nach cgm 394 (saec. xi) von Valentin Hartung (gen. Pacaeus) in Dillingen besorgt; s. Amelungk (1899) 4 Anm. 2. Einführendes zu den älteren Ausgaben auch bei Bihlmeyer (1956) xxxii-xxxiv; Ehrman (2003) 213-215.

6 Sondern aus dem späten 4. Jahrhundert. Die maßgeblichen Studien dazu sind nach Amelungk (1899) Weijenborg (1969), der eine Verbindung mit Evagrius Ponticus, und Hagedorn (1973), der einen „arianischen“ Kontext für die späteren Fälschungen vermutete. Weitere Informationen bei Schoedel (1990) 29-32; Löhr (2009) 105f.; Theobald (2016) 255f. u. Anm. 40.

7 Die kürzeste, auch „kürzere“ genannte Rezension ist lediglich eine nur auf Syrisch erhaltene Epitome von IgnPol, IgnEph und IgnRom mit einem Abschnitt aus IgnTrall; s. Schoedel (1990) 2324. Sie basiert auf der sog. „mittleren“ Version, stellt also keinen eigenen Überlieferungsstrang dar. Jene „mittlere“, das von Eusebius bezeugte Siebener-Corpus enthaltende Version kann noch am ehesten für sich den Anspruch auf „Ursprünglichkeit und Echtheit“ (Prostmeier [2002] 347) in dem Sinne erheben, dass Ignatios selbst es spätestens gegen Ende seines Lebens (d. h. gegen Ende der Herrschaftszeit Trajans) abgefasst haben könnte. (Man kann das Siebener-Corpus aber auch in dem Sinne als „ursprünglich und echt“ verstehen, dass es in dieser Form zwischen etwa 160 und 180 verfasst und zusammengestellt und unter dem Namen des Ignatios in Umlauf gebracht wurde, zusammen mit dem interpolierten Polyc, um die Glaubwürdigkeit des Konstrukts zu untermauern.) Die in diesem Siebener-Corpus überlieferten Texte stimmen auch in textlicher Hinsicht eher mit patristischen Zeugnissen überein als die in der längeren Rezension überlieferten Texte. Basierend auf hauptsächlich einer einzigen Handschrift, Florenz, BML, Plut. 57.7 (saec. xi), wurde es zuerst von Isaak Voss (1646) ediert. Darin nicht enthalten war IgnRom, für den in Paris, BnF, grec 1451 (= Parisiensis-Colbertinus 1451; saec. xi), erstmalig ediert von Thierry Ruinart (1689), ein eigener Überlieferungsstrang vorlag. Theodor Zahn (1873) und Joseph [nicht John, wie Löhr (2009) 107] B. Lightfoot (21889) lieferten die grundlegenden Argumente für die Rückführbarkeit des SiebenerCorpus auf den „historischen“ Ignatios; gegen Lightfoot s. aber bereits Killen (1886); ausführlich zum pseudepigraphischen Charakter des Corpus sowie zu den Interpolationen in Polyc s. Lechner (1999) 6-65; Theobald (2016) 252-331.

8 Es ist hier nicht möglich, alle relevanten Beiträge aufzulisten. Festzuhalten ist, dass bereits sehr früh, etwa mit den Magdeburger Centuriatoren (Flacius [1559] 165), gefolgt von Dallaeus (1666), Echtheitskritik einsetzte. Voss (1646) deutet in seiner Einleitung an, dass viele nicht nur bezweifel- 
auch die Reaktion darauf) vor allem kontroverstheologisch motiviert, wobei dieser Aspekt bis heute eine Rolle spielt, ${ }^{9}$ so wurden nach und nach verstärkt literarkritische und dogmen- und ideengeschichtliche Argumente ins Feld geführt, um den pseudepigraphischen Charakter des Corpus zu erweisen. Betont wurde die literarisch-artifizielle Konstruktion der Sammlung, ${ }^{10}$ die in den Briefen vorausgesetzte und theologisch begründete triadische Ämterhierarchie, ${ }^{11}$ die Präsenz von Polemik

ten, dass die von ihm edierten Briefe von Ignatios verfasst worden waren, sondern dass Ignatios überhaupt je Briefe verfasst habe. Man könnte es beinahe für eine Ironie der Vorsehung halten, dass gerade in den Vorsatzblättern eines in der Bibliothek des Klosters von Mar Saba in Jerusalem enthaltenen Exemplars von Voss' Ausgabe eine Handschrift entdeckt wurde, von der behauptet wird, sie sei der Text eines apokryphen gnostischen Markusevangeliums; s. dazu jetzt Burke (2013); Huller und Gullotta (2017). Barnes (2008) 120 Anm. 8 verwirft die „Entdeckung“ als einen Schwindel (engl. „hoax“), was angesichts ihrer komplexen Umstände und Hintergründe (s. Huller und Gullotta [2017]) etwas vorschnell erscheint.

9 Bei den Magdeburger Centuriatoren und Dallaeus, Echtheitsgegnern, wird dies zwar nicht explizit gemacht. Der Zeitkontext der Ausgabe Voss' (1646) sollte allerdings zu denken geben. Brent (2009) 1-2 erinnert daran, wie politisch geladen die Rolle Ignatios' als eines Verteidigers hierarchischepiskopaler Amtsstrukturen in einer Epoche war, in der nicht nur Erzbischöfe wie William Laud (1645), sondern sogar Könige wie Karl I. (1649) auf dem Schafott endeten. Der Ausspruch Jakobs I., „no bishop, no king“, wäre im selben Zusammenhang zu verstehen (auch wenn er zunächst speziell auf die schottische Situation abgezielt haben mag). Aber auch noch Brent selbst bringt zumindest im Untertitel seines Ignatios-Buches (2009) den Märtyrerbischof Ignatios mit „the origin of Episcopacy“ in Verbindung, wenngleich er es für sich verneint, ein kontroverstheologisches Interesse zu hegen. Auf römisch-katholischer Seite hat Gerhard Ludwig Müller ein Interesse angemeldet, das Zeugnis der Ignatianen für die Legitimierung bestehender Amtsstrukturen in Anspruch zu nehmen; s. Ayán Calvo (2002), zitiert bei Theobald (2016) 256 Anm. 42; weitere Ausführungen zur frühneuzeitlichen Rezeption bei Barnes (2008) 119-122.

10 In einer der jüngsten Studien dieser Art etwa kommt Michael Theobald (2016) 252-331 zu dem Schluss, dass die „sieben Ignatianen ... - literarisch hochartifiziell - am Corpus Paulinum Maß nehmen“ (ebd. 12). Der Verfasser lasse seinen „Helden, den sonst unbekannten Bischof von Antiochia, erklären, er möchte ,auf den Fußspuren des Paulus erfunden werden' (IgnEph 12,2). Dass ihn der Autor der ,Ignatianen' von Antiochia aus durch Kleinasien und Makedonien ins römische Martyrium schickt, stellt ihn an die Seite des ,pastoralen“ Paulus“ (ebd.); s. auch ebd. 297-300 und bes. 300 (in Auseinandersetzung mit früheren Studien) das Argument, dass gerade „Künstlichkeit und Komplexität“ Pseudepigraphie indizierten. - Diese letztere Einsicht ist eine Weiterführung des Arguments für den pseudepigraphischen Charakter der Pastoralbriefe, wofür auch der von Theobald nicht berücksichtigte Beitrag von Brox (1976) aufschlussreich ist, der sich mit Ps.-Ignatios, Ad Mariam, auseinandersetzt, sowie die formkritischen Bemerkungen zu Ignatios bei Sieben (1978); zu Letzterem s. Lechner (1999) 24. 130-134. Weder Brox noch Sieben jedoch zogen aus ihren Beobachtungen Schlüsse in Bezug auf den pseudepigraphischen Charakter des Siebener-Corpus. - Zur Paulusnachahmung in den Ignatianen s. auch im vorliegenden Band den Beitrag von Thomas Johann Bauer („Ignatios alter Paulus?").

11 Diese weise, so wird auch in jüngeren Studien argumentiert, eher auf eine Zeit nach der Mitte des 2. Jahrhunderts hin; s. bereits Killen (1886), der in seiner Kritik an Lightfoot jedoch noch viel weiter geht (IgnRom eine Fälschung des 3. Jahrhunderts); dann Joly (1979), Gryson (1979), Schmithals (2009); zu Letzterem s. auch die Diskussion bei Theobald (2016) 309-314. 
gegen bestimmte, wenn auch nicht namentlich genannte Gegner, und Bezüge zu Autoren der Mitte und zweiten Hälfte des 2. Jahrhunderts, etwa Ptolemäus, dessen Lehren „Ignatios“ gekannt habe, ${ }^{12}$ Markion und Noët, von deren Aussagen einige in den offensichtlich anti-valentinianischen Argumenten des „Ignatios“ wiedererkennbar seien, ${ }^{13}$ und sogar Lukian von Samosata, dessen Darstellung des Peregrinus Proteus vom Motiv der Martyriumsbereitschaft in den Ignatianen beeinflusst gewesen sein könnte. ${ }^{14}$

Die von der Forschung vor allem der letzten zwanzig Jahre im ignatianischen Corpus gemachten Beobachtungen legen daher eine Reihe möglicher Szenarien für die Entstehung des Corpus als eines pseudepigraphischen Kompilats in der zweiten Hälfte des zweiten Jahrhunderts nahe, wobei als terminus ad quem die frühen 180er Jahre anzusetzen wären, der Zeitraum in dem Irenäus sie erstmals erwähnte. ${ }^{15}$ Ebenfalls anzunehmen wären in diesem Fall ihr Entstehen nach dem Tod Polykarps (wohl 156 ${ }^{16}$ ) sowie Interpolationen an entsprechenden Stellen im Polykarpbrief. ${ }^{17}$ Ihr starker „Rombezug“ (Theobald), ${ }^{18}$ die Auseinandersetzung mit der um diese Zeit in Rom aktuell werdenden Amtsfrage (Monepiskopat) in Verbindung mit der Gottesfrage (Monarchianismus) und der Frage zur Haltung gegenüber dem Martyrium (vor allem auch angesichts des kurz vorher erfolgten Martyriums Polykarps), und schließlich die Verbindung mit Smyrna (Polykarp, Noët) lassen ein Entstehungs-

12 So Barnes (2008), bes. 123-125, der infolgedessen die Ignatianen ,in the 140s“ datiert.

13 So Hübner (1997; 1999) und vor allem auch Lechner (1999). Lechner nimmt folglich ein Entstehen des Corpus in Kleinasien zwischen 165 und 175 an; zu Markion s. ebenfalls Lechner (1999) 27-36. Die möglichen Verbindungslinien zu Markion und Noët könnten auch an Rom als Abfassungsort denken lassen; s. Theobald (2016) 309-314 (starker „Rom-Bezug“) und 256 Anm. 43. Theobald verweist ebd. auf den Vortrag von Ferdinand Prostmeier, „Cui Bono? Ignatios von Rom“, der im vorliegenden Band mit enthalten ist.

$14 \mathrm{Zu}$ möglichen Verbindungslinien zwischen den Ignatianen und Lukians De morte Peregrini s. Lechner (1999) 27-36. 64-67; Zwierlein (2009) 183-237, bes. 200 Anm. 183 sowie, ebd. zitiert und diskutiert (und am Ende verworfen), Parvus (2008), der, eine Theorie von Joseph Turmel aus den 1920er Jahren aufgreifend, den Verfasser der Ignatianen mit einem „markionitischen“ (bzw. „apelleianischen“) Bischof, Theophorus, und zugleich mit Lukians Figur Peregrinus identifiziert; zu älteren Theorien in dieser Richtung s. bereits Bihlmeyer (1956) xxxiv. Gegen Brent (2006; 2009) argumentiert Thomas Lechner in seinem Beitrag zum vorliegenden Band („Ignatios von Antiochia und die Zweite Sophistik“), dass die in den Ignatianen enthaltenen Anklänge zur Zweiten Sophistik kaum als Belege für eine Datierung des Corpus ins frühe zweite Jahrhundert herhalten könnten.

15 Iren., $a d v$. haer. 5,28,4; zitiert wird dort IgnRom 4,1. Die Stelle wird auch von Eus., hist. eccl. 3,36,12 zitiert; zur Datierung der Irenäusstelle s. Theobald (2016) 312-314. 330f.

16 Dehandschutter (2009) 130 mit Anm. 1; zitiert bei Theobald (2016) 312.

17 Insbesondere in Polyc 1,1-3 und 13,2; dazu im Detail Joly (1979) 17-37; Hübner (1997) 48-50; Lechner (1999) 6-65; Theobald (2016) 317-325; dagegen Brent (2009) 144-148.

18 Theobald (2016) 312-314. 330f. (vgl. oben Anm. 13); s. auch Lampe (2003) 88f., der zwar die Authentizität des Corpus anzunehmen scheint, es aber, von seiner Rezeption her denkend, in einen Kontext in Rom zwischen 160 und 180 stellt. 
datum zwischen den späten 160er und 170er Jahren nicht länger als eine Extremposition, sondern durchaus plausibel erscheinen. ${ }^{19}$

Michael Theobalds neue Studie ist für den vorliegenden Beitrag insofern von besonderer Relevanz, als sie die Feststellung des pseudepigraphischen Charakters der Siebener-Sammlung und der Verwendung und Adaption von Polykarps Brief an die Philipper zur Untermauerung des Konstrukts zum Ausgangspunkt nimmt, eine besondere Eigenschaft des Corpus herauszuarbeiten, nämlich seine Affinität zum Corpus Paulinum sowie $\mathrm{zu}$ den Pastoralbriefen. ${ }^{20}$ Intention des vorliegenden Beitrags ist es, ausgehend von Theobalds Untersuchungen bestimmte Aspekte des paulinischen Briefstils ${ }^{21}$ auch an den Ignatianen aufzuzeigen und interpretierend nachzuvollziehen und damit einen wenigstens bescheidenen Beitrag zum besseren Verständnis der Pragmatik letzterer zu leisten. Bei den gewählten Motiven handelt es sich um Aspekte der Thematisierung und (im Zuge derselben) Theologisierung der (brieflichen) Kommunikation selbst, insbesondere um das Inbeziehungsetzen der Motive Reden und Zureden, Hören, Gehorchen, Zustimmen, Übereinstimmen und Schweigen. ${ }^{22}$ Auch wenn das Folgende in vielem skizzenhaft bleiben muss, hofft es doch, zumindest in einigen Ansätzen die Bedeutung dieser Motive und ihrer Kombination in den Ignatianen erläutern zu können, und zwar sowohl im Hinblick auf ihre kommunikationsstrategische Funktion, als auch auf ihre Rolle bei der Formulierung einer ganz eigenen „ignatianischen“ Theologie.

Gehen wir zunächst von Theobalds Beobachtungen aus. Theobald beginnt mit der Frage, wie die Sieben-Zahl der im Corpus enthaltenen Briefe zu deuten sei: ${ }^{23}$

19 Gegen Barnes (2009) 122f., der meint, die Alternative vor 120 und nach 160 sei ein falsches Dilemma, und 140 als eine Mittelposition vorschlägt. Damit ignoriert Barnes völlig die positiven Indizien, die für eine späte Datierung sprechen. Lechner (1999) 306 grenzt den Zeitraum auf 165-175 ein und denkt an „Kleinasien“ als Entstehungsgebiet des Corpus. Theobald (2016) 312-331 hat diese These weiter verfeinert (s. auch oben Anm. 13).

20 Theobald (2016) 258 spricht hier insbesondere von der „Intertextualität der Ignatianen mit dem Corpus Paulinum“. Affinitäten zwischen Ignatianen und Paulinen wurden schon in früheren Studien wahrgenommen, etwa von Mitchell (2006) und bereits von Bultmann ([1953] 1967) und Rathke (1967), jedoch nicht mit Blick auf die Ignatianen als eines pseudepigraphischen Corpus der zweiten Hälfte des 2. Jahrhunderts.

21 Generell zum paulinischen Briefstil im Kontext der Epistolographie der frühen Kaiserzeit s. Bauer (2011) 101-105. 396-404; speziell zur hier angerissenen Thematik s. auch den Beitrag von Thomas Johann Bauer im vorliegenden Band („Ignatios - alter Paulus?“).

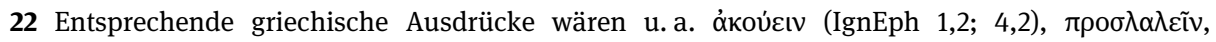

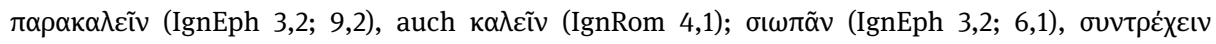

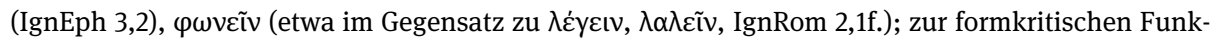
tion von $\pi \alpha \rho \alpha \kappa \alpha \lambda \varepsilon \dot{\varepsilon} \omega$ s. bereits Sieben (1978), ausführlich auch Lechner (1999) 21-35. 130-135; zur vielfältigen Rolle des Schweigens (im Hinblick auf seine Bedeutung in Gnosis, Prophetie sowie in der Herausbildung von Gotteslehre, Schöpfungslehre, Christologie und Anthropologie) s. Schoedel (1990) 48. 110-112. 144f. 164f. 207-210. 274f. 310.

23 Theobald (2016) 259. 
Nehme man an, die Sammlung sei ein pseudepigraphisches Artefakt, so werde sie wohl kaum auf Zufall beruhen. ${ }^{24}$ Ihr komme deutlich eine symbolische Bedeutung zu. Nach IgnRom 4,1 betone „Ignatios“, sein Schreiben sei an „an alle Ekklesien“

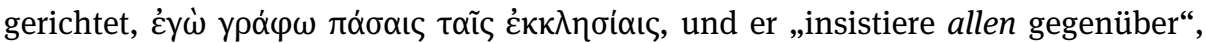
$\dot{\varepsilon} \nu \tau \dot{\varepsilon} \lambda \lambda \circ \mu \alpha \iota \pi \tilde{\alpha} \sigma \mathrm{t}$, dass er freiwillig das Martyrium auf sich nehmen wolle, sofern nur seine Adressaten ihn nicht davon abhielten.

Ehe wir mit dem Referat von Theobald fortfahren, eine kurze Bemerkung zum

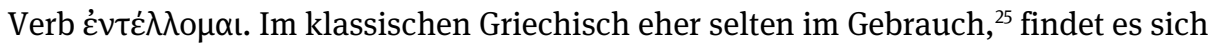
häufiger in jüdischen Autoren (LXX, Philo, Josephus) und auch im NT, wo es üblicherweise als „,anordnen“ oder „einschärfen“ (von Gesetzen und Geboten) übersetzt wird, ${ }^{26}$ synonym zu $\kappa \varepsilon \lambda \varepsilon v ́ \omega / \kappa \varepsilon \lambda \varepsilon v ́ o \mu \alpha$. Diese Bedeutung passt hier aber weniger, weil Ignatios, streng genommen, ja nichts anordnet, sondern eher seinen Adressa-

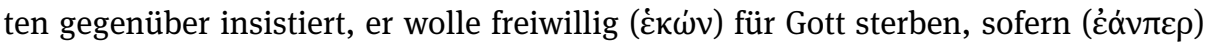
sie ihn nicht daran hinderten. Man könnte es lediglich so interpretieren, dass Ignatios seinen Adressaten „befiehlt“, ihn nicht vom freiwilligen Martyrium abzuhalten. Dies stünde einerseits in einem interessanten Kontrast zu IgnEph 3,1, wo Ignatios seinen Adressaten gegenüber ausdrücklich versichert, er wolle keine Befehle erteilen, oủ

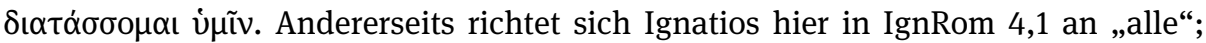
d. h. der Satz könnte als Formulierung eines allgemeinen, an die ganze Kirche ergehenden Befehls verstanden werden: „Hindert niemanden am freiwilligen Martyrium!“ Beide Aspekte sind interessant im Hinblick auf die Art und Weise wie „Ignatios“ mit seinen Adressaten zu kommunizieren versucht. Einerseits ist er plädierend und beteuernd und vermeidet ausdrücklich befehlende Wendungen, andererseits lässt er eine unterschwellige Kompromisslosigkeit und Härte spüren, wenn es darum geht, seinen Standpunkt zu vertreten, nämlich sein „Recht“, als Märtyrer sterben zu dürfen, ohne durch ein aus seiner Sicht falsches und irregeleitetes Wohlwollen davon abgehalten zu werden.

Da der Satz in IgnRom steht, ist Rom hier also offensichtlich als in dem Wort „alle“ mit eingeschlossen zu verstehen. Ignatios denkt hier an die ganze Kirche, in Ost und West. Bereits in IgnRom 2,2 hatte er auf sich selbst, weit ausholend und

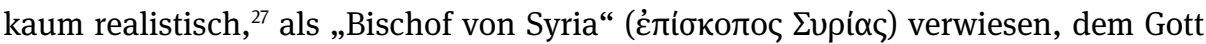

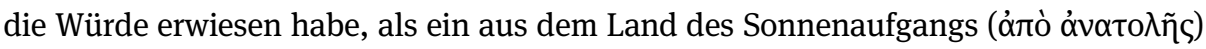

24 IgnEph 20,1 und IgnPol 8,1 widersprächen dem nicht; denn wenn Ignatios (,so Gott will“, $\theta \varepsilon \dot{\lambda} \eta \mu \alpha \tilde{\eta})$ einen zweiten Brief an die Epheser ankündige, so imitiere er damit lediglich Paulus (s. Theobald [2016] 304 zu Parallelen in Eph, Kol, 1Tim 1,4). Wenn er bedaure, er habe nicht allen Gemeinden in Kleinasien schreiben können, so übertrage er diese Aufgabe Polykarp, dessen Polyc ja eine wichtige Rolle für die Legitimierung des Siebener-Corpus spielen sollte.

25 Bzw. eher im Aktiv verwendet (s. etwa LSJ).

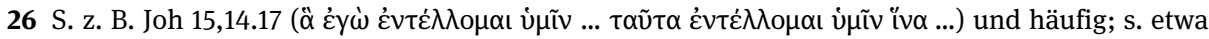
Mt 4,6; 17,9; 19,7; 28,20; Mk 10,3; 13,34; Lk 4,10; Apg 1,2; 13,47.

27 Zur Diskussion dieser Frage s. die oben Anm. 2 zitierte Literatur. 


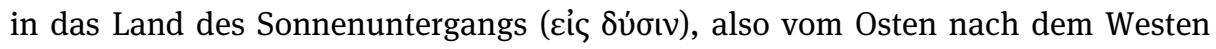
„Übersandter“ ( $\mu \varepsilon \tau \alpha \pi \varepsilon \mu \psi \alpha \dot{\alpha} \mu \varepsilon v o \varsigma)$ erfunden worden zu sein. Schön, d. h. angemessen, passend ( $\alpha \alpha \lambda o ́ v$ ), sei es, aus der Welt hinaus und auf Gott hin unterzugehen, um auf ihn hin aufzugehen. ${ }^{28}$ Die Siebener-Zahl, so Theobald, steht hier also für Ganzheit und Einheit, ähnlich wie auch in Offb 2 f. ${ }^{29}$

Auch dass sechs der Briefe an Kirchen adressiert seien und der siebte an eine Einzelperson, Polykarp, die zudem einer jener bereits angeschriebenen Kirchen vorgestanden sei, so Theobald, nämlich Smyrna, erkläre sich dadurch, dass eben sieben Briefe geschrieben werden mussten, um die Zahl vollzumachen. ${ }^{30}$ Damit sei allerdings nicht gemeint, dass die Anordnung der Briefe im übrigen ohne Plan sei bzw. dass die planmäßige Anlage des Corpus dem Zwang, sie partout in ein Siebener-Schema zu pressen, zum Opfer gefallen sein könnte. Die von Eusebius bezeugte Abfolge (IgnEph, IgnMagn, IgnTrall, IgnRom, IgnPhld, IgnSm, IgnPol), von der die der im Mediceo-Laurentianus überlieferten griechischen „mittleren“ Rezension (IgnSm, IgnPol, IgnEph, IgnMagn, IgnPhld, IgnTrall, IgnRom) nur geringfügig abweiche, zeuge vielmehr von einer strengen Planung. ${ }^{31}$ Sämtliche Versionen der Abfolge, die sich in den Zeugen der mittleren Rezension finden ließen, lieferten Indizien, die sie als voneinander abhängig erwiesen. IgnRom nehme in allen von ihnen eine zentrale Stellung ein, entweder in der Mitte oder am Ende der Reihe. Dementsprechend (in ihrer relativen Position zu IgnRom) stünden auch IgnSm und IgnPol entweder am Anfang oder am Ende der Reihe. ${ }^{32}$ Dabei scheine die von Eusebius bezeugte Version mit IgnRom in der Mitte und IgnSm und IgnPol am Ende die ursprünglichere zu sein, so Theobald weiter. Sie reflektiere das Itinerar des Ignatios in der intendierten Ordnung. Mit IgnSm und IgnPol stünden die beiden am stärksten individualisierten Briefe am Ende, IgnPol gewissermaßen als mögliches Pendant zum interpolierten Polyc, der der gesamten Sammlung zur besseren Legitimation beigefügt werden konnte. IgnRom als der wohl provokanteste und signifikanteste Brief der Sammlung stehe in der Mitte, IgnEph, das längste und programmatischste Schreiben, durch dessen Vermittlung alle übrigen Briefe zueinander in Bezug gebracht werden konnten, am Anfang. ${ }^{33}$

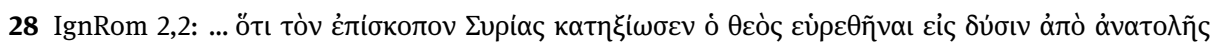

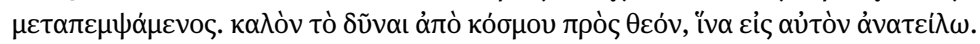

29 Dazu Theobald (2016) 260 bes. Anm. 54; s. ebd. auch zur Herkunft der Siebener-Zahl aus 4Makk, bes. 4Makk 14,7f.

30 Theobald (2016) 260. Auch hier könnte man erneut daran denken, dass Polykarp durch Polyc ja auch die Aufgabe zufällt, die Authentizität des Corpus zusätzlich zu untermauern.

31 Ausführlich dazu Theobald (2016) 262-270.

32 Je nachdem, ob die Sammlung gemäß dem Itinerar oder aus der Perspektive Polykarps (auch gemäß Polyc 13) geordnet worden sei; s. Theobald (2016) 271. 274.

33 Theobald (2016) 274. 
Von IgnEph ausgehend, lasse sich auch die „Geschichte“ des Ignatios, seine „Story“, wenn man so will, in stilisierter Form aus den Briefen rekonstruieren. ${ }^{34}$ Ignatios befinde sich auf dem Weg von Antiochia nach Rom. ${ }^{35}$ In Philadelphia habe er zum ersten Mal Halt gemacht. ${ }^{36}$ Während des zweiten Halts in Smyrna ${ }^{37}$ habe er IgnEph, IgnMagn, IgnTrall und IgnRom verfasst, ${ }^{38}$ während des dritten Halts, in Troas, IgnPhld, IgnSm und IgnPol..$^{39}$

Diese „Geschichte“ wie auch das Itinerar klingen an Paulus an und enthalten Paulus imitierende Elemente, weichen aber auch immer wieder von Paulus ab.

IgnRom 1,1 etwa bezeichne sich „Ignatios“ als „in Christus Jesus gebunden“,

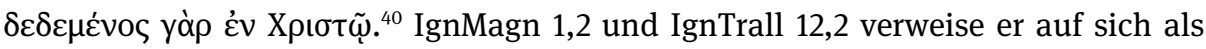

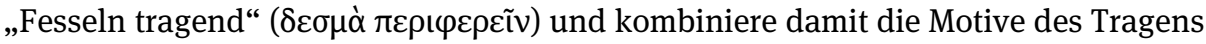
(des Leidens) Christi (vgl. Paulus, 2 Kor 4,10) und des Gebundenseins. ${ }^{41}$ Das Motiv

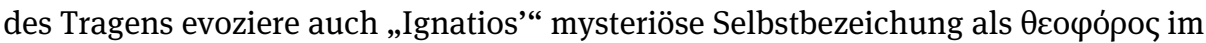
Präskript eines jeden der sieben Briefe. ${ }^{42}$ Das Itinerar, von Antiochia nach Rom, erinnere, so Theobald weiter, an die Schilderung der Paulusreisen in der Apostelgeschichte.

Freilich werde Ignatios in den Ignatianen weder als ein Nachfolger des Paulus (als Apostel) noch wie Titus und Timotheus in den Pastoralbriefen als ein von Paulus eingesetzter bzw. autorisierter Gemeindeleiter (Bischof) aufgebaut; sondern seine Autorität, die ja als eine universale (also nicht ortsbischöfliche) konzipiert werde, resultiere vielmehr aus seiner Nachahmung des Paulus in einer ganzen Reihe von verschiedenen Beziehungen, darunter etwa seine Reise von Antiochia nach Rom, seine Abfassung eines umfassenden, paulinisch beeinflussten Brief-Corpus, und sein Martyrium in Rom..$^{43}$ Dass sein Martyrium anderer Art als das des Paulus sei, setze

\footnotetext{
34 Theobald (2016) 275.
}

35 IgnRom 4,1; 2,2 (Ignatios Bischof); IgnEph 12,1, IgnTrall 3,3, IgnRom 4,3 (verurteilt); IgnPhld 1,1, IgnPol 8,1 (auf dem Landweg über Kilikien, dann weiter durch Asien und hinüber nach Makedonien); IgnRom 5,1 (unter Bewachung).

36 IgnPhld 1,1.

37 IgnSm 1,2f. und 2,1 (Empfang der Gesandtschaften aus Ephesus, Magnesia und Tralles).

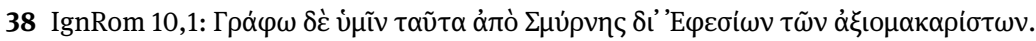

39 IgnPhld 11,1; IgnSm 12,1 (Gruß aus Troas); IgnPol 8,1 (plötzliche Abreise von Troas nach Neapolis; deswegen keine weiteren Briefe; Auftrag an Polykarp, diese Aufgabe zu übernehmen).

40 Theobald (2016) 286; vgl. Paulus, Phil 1,7; Phlm 1,1; ausserdem Eph 3,1; 4,1.

41 Theobald (2016) 286f.

42 Theobald (2016) 295f. mit Anm. 163; Schoedel (1990) 79-81; Brent (2009) 151-158.

43 Dazu dass Ignatios' Autorität in den Briefen gerade nicht episkopaler Art sei, s. Theobald (2016) 294. Bereits in IgnEph 1,1 etwa finden sich mehrere der genannten Motive akkumuliert: Ignatios kommt von Syria her (árì $\Sigma v \rho i \alpha \varsigma)$ und ist auf dem Weg nach Rom, um dort mit den wilden Tieren zu

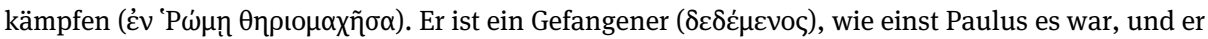

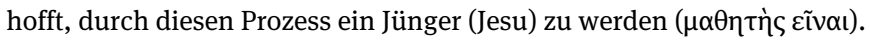


überdies gewisse Entwicklungen des christlichen Martyriumsgedankens im 2. Jahrhundert voraus. ${ }^{44}$

Grundlegend freilich bleibe bei all diesen verschiedenen Aspekten die eine Tatsache, dass der Zugang zu Ignatios über das Brief-Corpus erfolge, das ganz wesentlich das Vorbild eines Corpus Paulinum voraussetze ${ }^{45}$ wie auch das der Pastoralbriefe, die der Verfasser der Ignatianen offensichtlich nicht nur kenne, sondern an denen er sich auch literarisch orientiere. ${ }^{46}$

Um die hierbei relevanten und in den Ignatianen angewendeten Techniken brieflicher Kommunikation $\mathrm{zu}$ illustrieren, diskutiert Theobald drei Abschnitte, IgnEph 3,1f., IgnTrall 3,3 und IgnRom 4,1.3, deren Motive sich gegenseitig erhellen. ${ }^{47}$ IgnEph 3,1f., das unmittelbar auf die Eröffnungsabschnitte folgt, ${ }^{48}$ betont, „Ignatios“ wolle seinen Adressaten keine Befehle erteilen, als ob er groß jemand sei [sc. dem

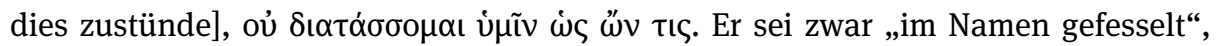
$\delta \varepsilon ́ \delta \varepsilon \mu \alpha \iota$ ćv $\tau \tilde{\omega}$ óvó $\mu \alpha \tau$, aber „noch nicht in Jesus Christus vollendet“, oű $\pi \omega$

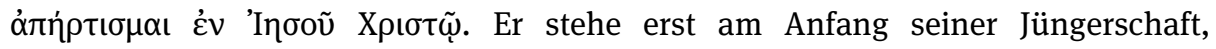
$\mu \alpha \theta \eta \tau \varepsilon \dot{\varepsilon} \sigma \theta \alpha$, und rede mit ihnen, seinen Adressaten, $\pi \rho \circ \sigma \lambda \alpha \lambda \tilde{\omega} \dot{v} \mu \tilde{v} v,{ }^{49}$ als seinen Mitjüngern, $\dot{\omega} \varsigma \sigma v v \delta ı \delta \alpha \sigma \kappa \alpha \lambda i \tau \alpha ı \varsigma ~ \mu o v$. Eigentlich, fährt er fort, müssten sie ihn mit

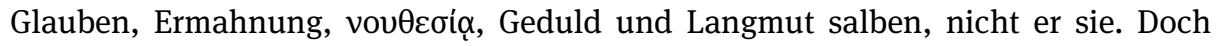

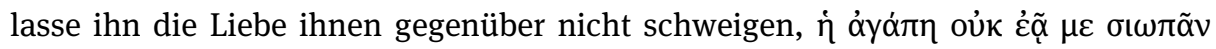

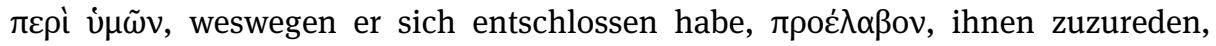

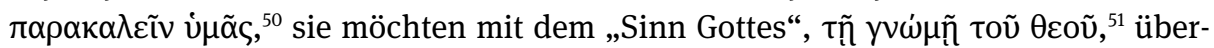
einstimmen, so wie Christus des Vaters Sinn sei und die Bischöfe, die bis an die

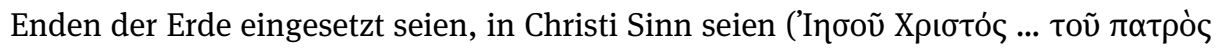

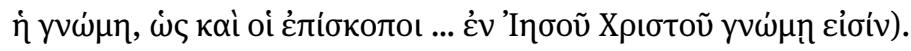

44 Theobald (2016) 289 sowie ebd. 281-283 mit Verweis auf einen möglichen Einfluss des Martyriumsgedankens in 4Makk; s. dazu auch Barnes (2010) 1-43, bes. 12-14, der in Bezug auf diese Möglichkeit allerdings Skepsis anmeldet. Barnes’ Aussagen zur Datierung der Ignatianen ebd. sind übrigens mit Vorsicht zu genießen; s. dazu Genaueres im Detail bereits oben unter Anm. 19 zu Barnes (2008).

45 Theobald (2016) 297.

46 Theobald (2016) 299, mit Verweis auf Merz (2004) 182f.

47 Theobald (2016) $290 f$.

48 S. dazu oben Anm. 40.

49 Das hier verwendete Verb, $\pi \rho \circ \sigma \lambda \alpha \lambda \varepsilon \tilde{v}$, bedeutet eigentlich „mit jemandem reden“. Es ist von dem in Abschnitt 2 folgenden $\pi \alpha \rho \alpha \kappa \alpha \lambda \varepsilon \tilde{v}$ scharf zu unterscheiden.

50 Zur formkritischen Bedeutung der Verwendung von $\pi \alpha \rho \alpha \kappa \alpha \lambda \varepsilon i ̃ v$ an dieser Stelle nicht nur für IgnEph, sondern für das ganze ignatianische Brief-Corpus s. Sieben (1978) 8f., wo auch der Kontrast

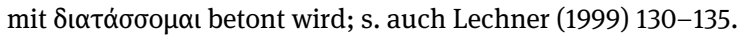

51 Die Übersetzung von yvú $\mu \eta$ mit „Sinn“ erfolgt hier nach Lindemann u. Paulsen (1992). Gemeint ist hier aber nicht nur ein subjektiver Zustand, sondern eine objektive Gegebenheit. Es wäre also vielleicht besser mit „Lehre“ zu übersetzen; s. dazu unten Anm. 63 und 64. 
Diese letzten Zeilen eröffnen einen Blick auf den „harten“, „dogmatischen“ Kern der Botschaft von IgnEph, der in den auf 3,2 folgenden Abschnitten noch stärker hervortreten wird. Aber hier in IgnEph 3,1-2 steht stattdessen der Wille zur Briefkommunikation im Vordergrund mit Motiven wie „miteinander reden“ ( $\pi \rho \circ \sigma \lambda \alpha \lambda \varepsilon \tilde{v}$ ),

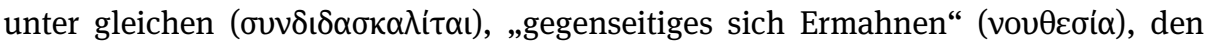
Adressaten „zureden“ ( $\pi \alpha \rho \alpha \kappa \alpha \lambda \varepsilon \tilde{v} \nu)$ mit der dezidierten Absicht ( $\pi \rho \circ \varepsilon \dot{\lambda} \alpha \beta \circ)$ ), sie zu überreden und zu überzeugen.

Es gilt, beide Seiten dieser, wie es scheint, rhetorischen Strategie im Auge zu behalten. Die beteuernden, plädierenden Elemente scheinen dazuhin angelegt zu sein, zunächst kommunikativ zu den Adressaten durchzudringen. Es handelt sich hier nicht um den dogmatischen Kern der Botschaft, die vermittelt werden soll. Letztlich scheint es „Ignatios“ durchaus um etwas zu gehen, das Entschiedenheit und Rigorosität abverlangt, um etwas nicht weiter Diskutables, das eingefordert wird wie ein Gebot oder Befehl..$^{52}$ Er selbst präsentiert sich ja als ein lebendiges Beispiel für diese Haltung, ein Vorbild für seine Adressaten. Doch, so beteuert er immer wieder, er wolle sich zurückhalten und seinen Adressaten nicht mit der Tür ins Haus fallen, in der Absicht eben, sie für sich und seine Botschaft zu gewinnen.

Die Durchsichtigkeit und Offensichtlichkeit dieser Doppelstrategie kann auch Verdacht erwecken. „Ignatios'“ Plädoyers und Beteuerungen wirken manchmal beinahe wie Drohungen.

IgnTrall 3,3 illustriert dies ein wenig. Hier bezieht sich Ignatios auf sich selbst und sagt, die Liebe, $\grave{\eta} \alpha \dot{\alpha} \alpha \dot{\pi} \eta$, von der auch oben im Kontext von IgnEph 3,1f. die Rede gewesen war, halte ihn davon ab, sich auf kräftigere Weise, „akzentuierter“

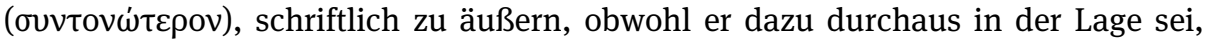

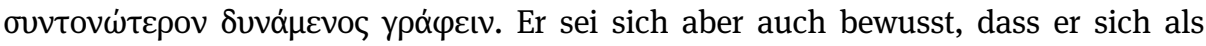

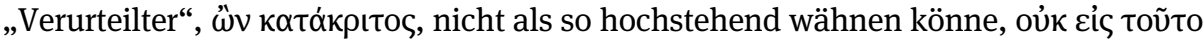
$\dot{\omega} \eta \dot{\theta} \eta \mathbf{v}$, dass er seinen Adressaten wie ein Apostel Befehle erteilen dürfte, $\dot{\omega} \varsigma$

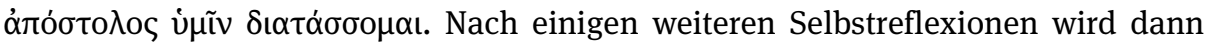
auch hier, IgnTrall 6,1, der eigentlich exhortative Abschnitt mit dem Verb $\pi \alpha \rho \alpha \kappa \alpha \lambda \tilde{\omega}$ eingeleitet: $\pi \alpha \rho \alpha \kappa \alpha \lambda \tilde{\omega}$ oũv $\dot{u} \mu \tilde{\alpha} \varsigma . .$. „So rede ich euch denn nun zu bzw. nicht ich, sondern die Liebe Jesu Christi.“" ${ }^{\circ 3}$

52 Im Corpus insgesamt lässt sich ein ganzer Komplex von „Anliegen“ ausmachen. In IgnRom etwa ist es unter anderem vor allem „Ignatios'“ Unmut (oder gespielter Unmut) über die von ihm befürchtete Bereitschaft seiner Adressaten (sc. der Christen von Rom), ihn vom Martyrium, das er entschlossen ist, auf sich zu nehmen, fernzuhalten (etwa IgnRom 4,1); in anderen Briefen ist es u. a. Polemik gegen Häretiker (IgnMagn 8,1; IgnTrall 6,1; 10; IgnPhld 2-3; IgnSm 2), Schismatiker (IgnEph 7,1; 16,1; IgnMagn 4; IgnSm 6-7), „Judaisierer“ (IgnMagn 8,1; IgnPhld 6) und vor allem auch gegen, Leute die die Autorität des Bischofs leugnen oder untergraben (IgnMagn 4 u. a.); zu letzterem Aspekt s. Trevett (1983).

53 Vgl. Theobald (2016) 290 Anm. 152. 
Aber der Kontrast ist deutlich. „Ignatios“ signalisiert, dass er es durchaus in

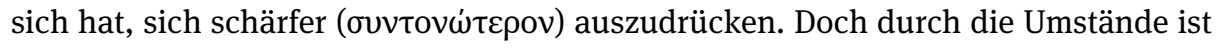
er gezwungen, vorsichtig zu sein, sich zurückzuhalten und den Konsens mit seinen Adressaten zu suchen. ${ }^{54}$

Die dritte von Theobald ausgewählte Stelle ist IgnRom 4,1.3:

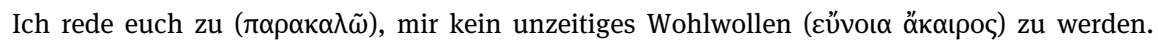
Lasst mich der wilden Tiere Fraß werden. Durch sie ist es möglich, Gott zu erlangen ... Nicht

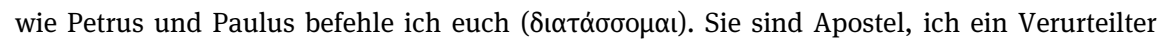

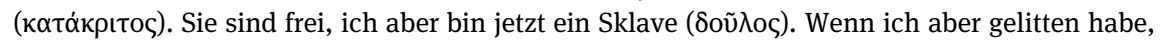

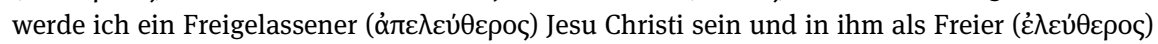
auferstehen. Jetzt lerne ich, als Gefangener ( $\delta \varepsilon \delta \varepsilon \dot{\varepsilon} \mu \varepsilon v o \varsigma)$ nichts zu begehren ( $\mu \eta \delta \dot{\varepsilon} v \dot{\varepsilon} \pi t \theta v \mu \varepsilon \tilde{v})$.

„Ignatios“, so Theobald, könne seinen Adressaten in seinem gegenwärtigen Status

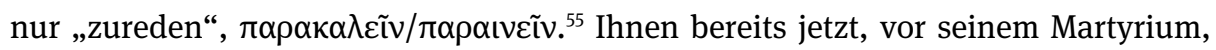

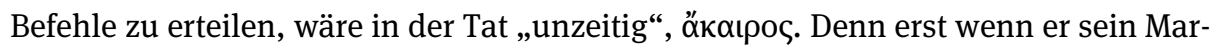
tyrium, das er ebd. in grausamen, körperbezogenen, aber auch auf eucharistische Symbole anspielenden Einzelheiten antizipierend beschreibt, ${ }^{56}$ erlitten hat, also von den wilden Tieren in der Arena aufgefressen worden ist, so wie Christus von den Gläubigen als Brot in der Eucharistie verzehrt wird, wird er ihnen wie ein Apostel

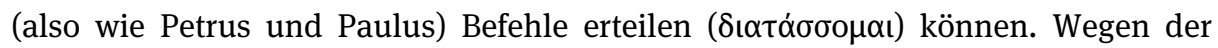
kausalen Beziehung zwischen Martyrium und apostolischer Autorität wäre es fehl am Platz, würden seine Adressaten in Rom versuchen, ihn vor seinem Schicksal zu bewahren. Sie wären ihm in diesem Fall ein „unzeitiges Wohlwollen“ (عűvoı $\alpha$

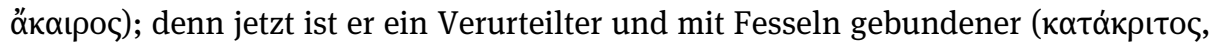

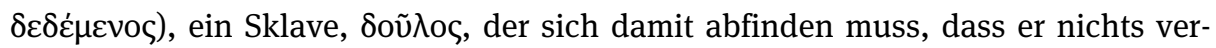

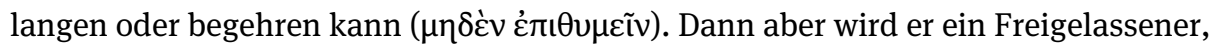
ja ein Freier sein, Christus gleich. Seine Paränese ist hier also antizipierend als eine Vorform seiner apostolischen Autorität konzipiert. Die kräftigere, schärfere Rede, auf die er in IgnTrall 3,3 anspielte, soll letztlich durch seine Tat, d. h. durch sein Leiden, sein Martyrium effektiv werden.

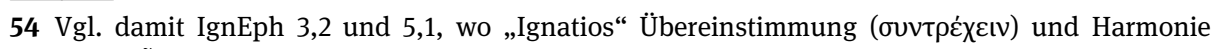
( $\sigma \dot{\mu} \mu \varphi \omega v \alpha$ ก̃) empfiehlt, oder IgnRom 2,2, wo er das Bild von der Gemeinde evoziert, die in Liebe ein

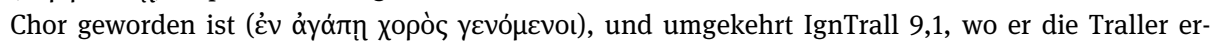
mahnt, sich einfach taub zu stellen gegenüber denen, die Spaltung propagieren ( $\kappa \omega \varphi \dot{\theta} \theta \eta \tau \varepsilon$ oũv), oder IgnEph 9,1, wo beschrieben wird, wie die Epheser sich gegen Irrlehrer ihre Ohren verstopfen ( $\beta \dot{\sigma} \sigma \alpha \nu \tau \varepsilon \varsigma \tau \grave{\alpha} \omega \tilde{\omega} \tau \alpha)$.

55 Theobald (2016) 295.

56 IgnRom 4,1: „Gottes Weizen bin ich und durch der wilden Tiere Zähne werde ich gemahlen,

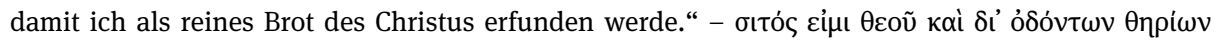

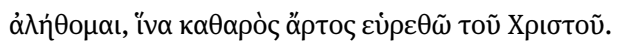


Entfernen wir uns von hier an von der Darstellung Theobalds und nehmen wir

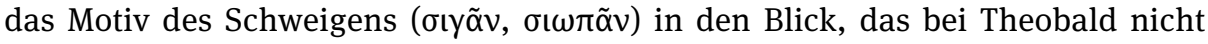
eigens zur Geltung kommt. In IgnTrall 3,3 hatte „Ignatios“ geschrieben, dass es die

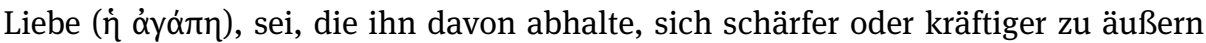

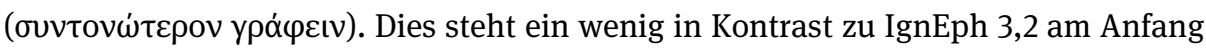
des Corpus, wo er betont, die Liebe (’ $\dot{\gamma} \gamma \alpha \dot{\alpha} \pi \eta)$ lasse ihn im Hinblick auf seine Adressaten im Gegenteil gerade nicht schweigen ( $\sigma \iota \omega \pi \tilde{\alpha} v)$, weshalb er sich vorgenommen

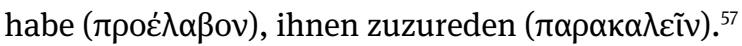

Der Gebrauch des Verbs $\pi \rho \circ \lambda \alpha \mu \beta \alpha \dot{\alpha} \omega \omega$ an dieser Stelle ist programmatisch und von hoher Signifikanz. „Ignatios'“ Entscheidung, sich sprachlich zu äußern, ist antizipatorisch motiviert, wie oben bereits im Zusammenhang mit IgnRom 4,1 kurz angedeutet. Die Tat, die Aktion, die seiner Rede Wirkkraft verleihen soll, nämlich das Martyrium, steht noch aus. Deshalb ist Schweigen, etwa in einem Bischof, höher zu schätzen als Reden, ${ }^{58}$ und für „Ignatios“ Zureden ( $\left.\pi \alpha \rho \alpha \kappa \alpha \lambda \tilde{\omega}\right)$ eher angebracht als Befehlen ( $\delta \iota \alpha \tau \alpha \sigma \sigma o \mu \alpha)$. Dennoch ist der vorausschauende und vorgreifende Entschluss, was die Philosophen $\pi \rho o ́ \lambda \eta \psi ı \varsigma$ nennen, ${ }^{59}$ bereits in dieser vorläufigen Situation das Wort zu ergreifen, und zwar schriftlich, mittels paränetischer Briefe, nicht nur gerechtfertigt, sondern geradezu ein Imperativ. „Ignatios“ fühlt sich geehrt ( $\left.\eta_{\xi} \iota \dot{\omega} \theta \eta v\right)$, und frohlockt darüber ( $\left.\alpha \gamma \alpha \lambda \lambda เ \tilde{\omega} \mu \alpha \iota\right)$, in dieser Weise auf seine Adressaten zugehen zu können in der Absicht, mit ihnen zu kommunizieren $(\pi \rho \circ \sigma o \mu \iota \lambda \tilde{\eta} \sigma \alpha \mathrm{l})^{60}$

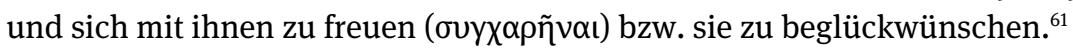

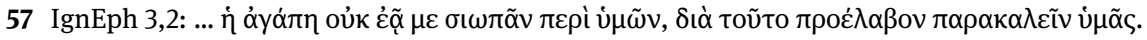

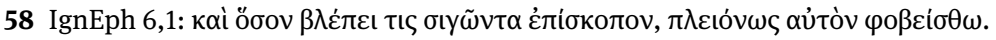

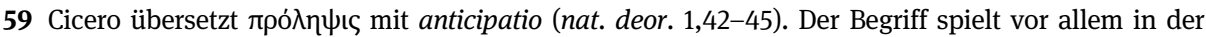
stoischen Erkenntnislehre und Ethik eine Rolle. Damit soll hier nicht gesagt sein, dass „Ignatios“ im

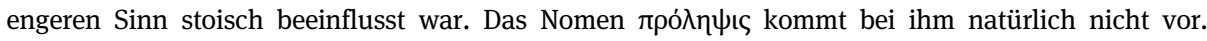
Bonhöffer (1911) hat jedoch auf einer etwas allgemeineren Ebene gewisse Überschneidungen zwischen Epiktet und „Ignatios“ im Gebrauch entsprechenden Vokabulars wahrgenommen, etwa IgnEph 7,2

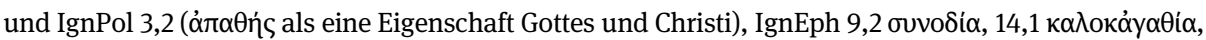

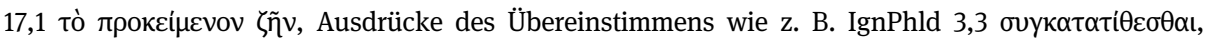

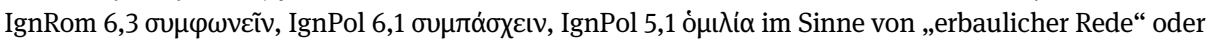
„Abhandlung“; IgnRom 4,1 غ̇кúv, „freiwillig“ und andere mehr. Den unter hellenistischen Philoso-

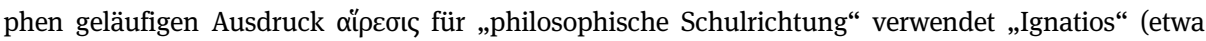
IgnEph 6,2; IgnTrall 6,1) bereits (als einer der ersten seines Zeichens) in seiner christlichen (peijorativen) Konnotation („Häresie“, „Abspaltung“, „Sekte“); vgl. Glucker (1978) 186 Anm. 63 und 205.

60 Angesichts von IgnPol 5,1, wo ò $\mu \lambda \lambda \dot{\alpha} \alpha$ roเعĩv erstmals im Sinne von „predigen“ verwendet wird,

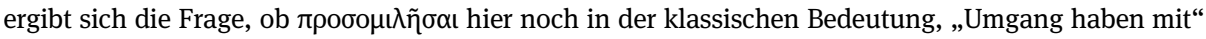
(vgl. etwa ò $\mu \lambda \varepsilon \varepsilon i v$ im 1Kor 15,33 zitierten, bei Menander überlieferten Sprichwort), oder schon in der neueren, auch von der Stoa, etwa Epiktet eingeführten Bedeutung, ,jemandem einen (erbaulichen oder philosophischen) Vortrag halten“, zu verstehen ist; s. Bonhöffer (1911) 122; vgl. Schoedel (1990) 124, „sich unterhalten“; Lindemann u. Paulsen (1992) 185, „zu euch zu reden“.

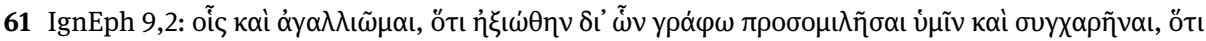

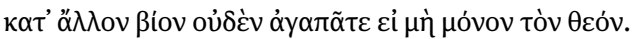


Die Forschung hat das Motiv Schweigen in den Ignatianen vornehmlich unter der Rücksicht möglicher Abhängigkeiten von gnostischen Quellen untersucht. ${ }^{62}$ IgnEph 6,1, wo vom Schweigen des Bischofs die Rede ist, das von einigen als Vor-

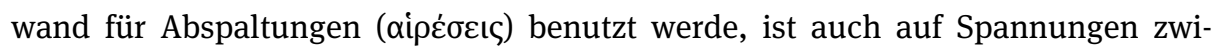
schen einerseits eher amtlich-hierarchisch und andererseits eher prophetischcharismatisch orientierten Gemeindemitgliedern gedeutet worden. ${ }^{63}$ Doch wird dem Schweigen in allen Briefen auch ganz einfach ein Eigenwert im Kontext der Briefkommunikation zugewiesen. Dass dies nicht ohne Spannungen geschieht, ist bereits erwähnt worden, im Hinblick auf IgnEph 3,2, wo „Ignatios“ schreibt, die Liebe ( $\dot{\eta} \alpha \dot{\gamma} \alpha \dot{\pi} \pi \eta)$ habe ihn einfach nicht schweigen ( $\sigma \iota \omega \pi \tilde{\alpha} v)$ lassen, weswegen er sich vorgenommen habe, seinen Adressaten wenigstens zuzureden ( $\pi \alpha \rho \alpha \kappa \alpha \lambda \varepsilon \tilde{v})$. Ziel dieser Kommunikationsstrategie ist Übereinstimmung in der Lehre und mit dem Magiste-

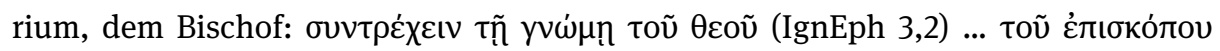
(ebd. 4,1). ${ }^{64}$ Das Ziel ist Harmonie, Gleichklang der Stimmen wie in einem Chor

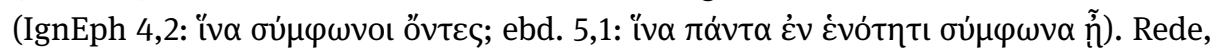
auch theologische Lehrrede und paränetische Rhetorik, wird hier also transzendiert auf die musikalische Stimme ( $\varphi \omega v \eta \dot{)}$ hin, in der der Inhalt nicht mehr bestimmt werden muss, da er nicht mehr strittig ist. Aber das ist noch nicht das Ende; denn IgnEph 6,1 folgt die Rede vom schweigenden Bischof, der aufgrund seines Schweigens umso mehr zu ehren sei. Er redet quasi durch sein Amt bzw. durch seine Tu-

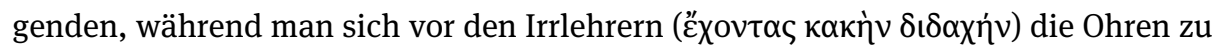

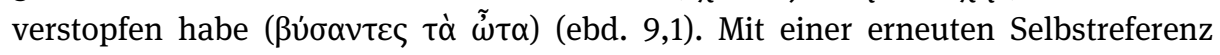
beschließt „Ignatios“ diesen Abschnitt: Er fühle sich geehrt, dass er sich schreibend

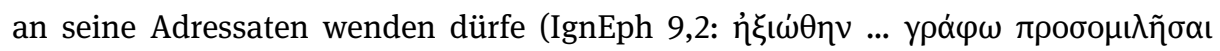

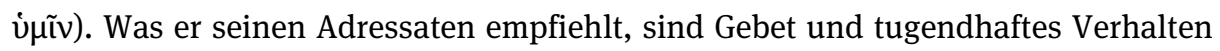
(besonders im Angesicht der Provokation der innerkirchlichen Gegner).

Der hierauf folgende Abschnitt (IgnEph 11-15) kulminiert erneut in einem Lob des Schweigens (IgnEph 15,1), das diesmal sowohl mit metaphysischen als auch mit schöpfungstheologischen und christologischen Elementen untermauert wird:

62 S. etwa Schoedel (1990) 158-170; Lechner (1999) 251-270 zum Oxymoron des Aufschreis (крауүп̃ऽ)

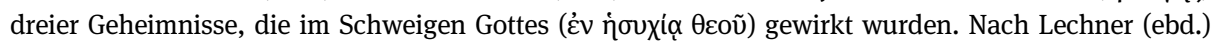
handelt es sich bei i̇ouxí $\alpha$ an dieser Stelle um einen valentinianischen Begriff.

63 S. dazu Trevett (1983) 9-16 mit Verweis auf IgnEph 6,2, IgnMagn 4, IgnTrall 7,2 u. a.; zum Aus-

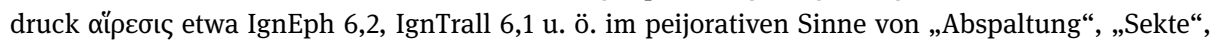

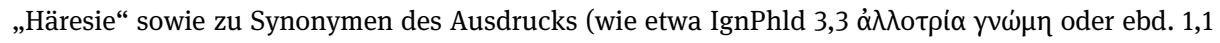

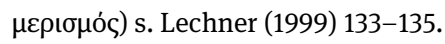

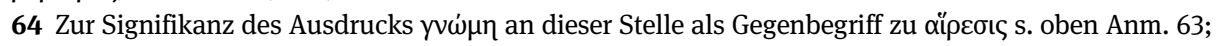
und vgl. auch oben Anm. 51 sowie unten Anm. 89. 
Besser ist es, zu schweigen und zu sein, als redend nicht zu sein. Angemessen ${ }^{65}$ ist das Lehren, wenn der Redende dabei etwas bewirkt und schafft. Nun aber gibt es nur einen einzigen solchen Lehrer, der „sprach und es wurde“ [Ps 32,9; 148,5]; und was er schweigend schuf, ist des Vaters würdig. ${ }^{66}$

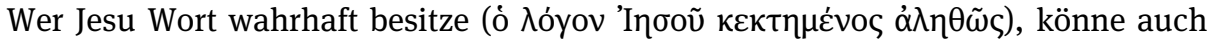

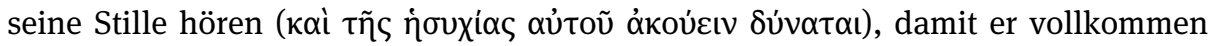
( $\varepsilon \dot{\lambda} \lambda \varepsilon ı \varsigma)$ werde. Er werde durch sein Handeln reden und an seinem Schweigen er-

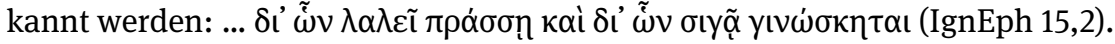

Hier nun also werden Reden, Schweigen, Hören, Handeln und Erkanntwerden in Beziehung gesetzt und mit Gott, dem Schöpfungsakt und Christus assoziiert. Zunächst wird der inhärente Wert des Redens allgemein infragegestellt: In der Wirklichkeit (Gottes) verankertes Schweigen ist besser als nicht auf Wirklichkeit gegründetes Reden. Dass mit Reden hier vor allem lehrendes Reden gemeint ist, ergibt sich

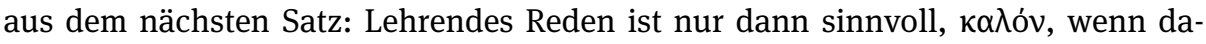
durch eine Wirkung erzielt, d. h. wenn dadurch etwas geschaffen wird, wenn es

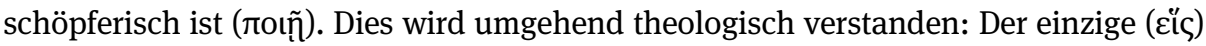
Lehrer von dem wirklich gesagt werden kann, dass durch sein Wort etwas geworden ist, und es wird hier sogar ein Psalmenzitat bemüht, ist Gott. Im Psalm ist es Jahwe, „der Herr“ (LXX: ò kúpıos). Hier ist es der Lehrer, Christus. Da seine Rede mit seinem Handeln identisch ist, d. h. sich in nichts anderem als in seiner Schöpfung manifestiert, ist es paradoxerweise nicht im üblichen Sinn ein Reden, sondern vielmehr ein Schweigen. An ihm wird es für das erkannt, was es ist, nämlich Handeln und Wirken Gottes, des Vaters selbst würdig, Offenbarung des in seinem Wesen Verborgenen. ${ }^{67}$

In IgnMagn und IgnTrall spielt die hier interessierende Thematik eine weniger ausgeprägte Rolle. Gegen Ende von IgnMagn, in Kapitel 14, wird den Adressaten nur knapp bescheinigt: „Da ich weiß, dass ihr von Gott erfüllt seid, habe ich euch nur

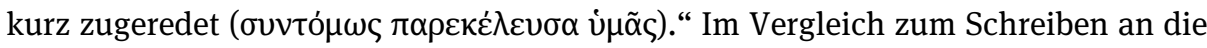
Epheser ist der Brief an die Magnesier in den Augen seines Verfassers also eher eine Epitome. Dennoch wird ihm dieselbe Funktion bescheinigt. Der Autor wendet sich „zuredend“, plädierend, an seine Adressaten, in der Absicht, sie von einer bestimmten Glaubenshaltung zu überzeugen.

In IgnTrall 5,1 taucht erneut das Motiv des Maßhaltens in diesem Unterfangen auf. „Ignatios“ erwähnt hier, dass er durchaus in der Lage wäre, seinen Adressaten

65 Ka入óv. Lindemann u. Paulsen (1992) 187 übersetzen „gut“. Doch s. IgnEph 14,1 die Rede von

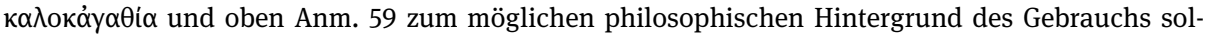
cher Ausdrücke.

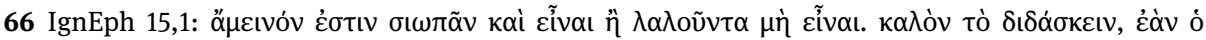

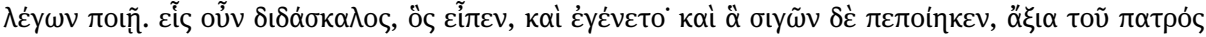
Éotiv.

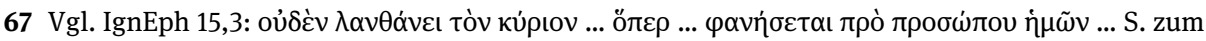

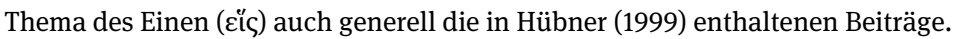


mehr über „die himmlischen Dinge“ ( an den oben erwähnten Stellen verschreibt er sich selbst Zurückhaltung, und zwar mit der Begründung: „Ich fürchte, ich könnte euch sonst als Unmündigen Schaden

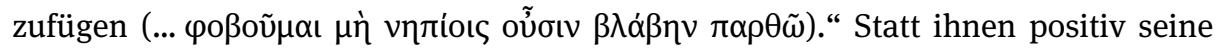
Lehre in ihrer Komplexität darzulegen, zieht „Ignatios“ es vor, sie vor den Lehren

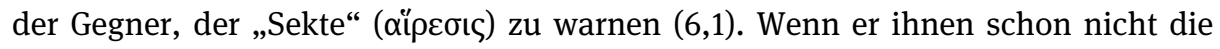
schwere, für sie unverdauliche Kost seiner Lehre verabreichen konnte, sollten sie sich wenigstens gegenüber den Gegnern taub stellen (9,1: $\kappa \omega \varphi \omega \dot{\theta \eta \tau \tau \varepsilon ~ o u ̃ v ~ . . .), ~ w i e ~ e s ~}$ ja auch die Epheser taten (s. IgnEph 9,1). Das sich taub Stellen gegenüber den Häretikern wird an diesen Stellen also beinahe wie ein Pendant zum „Hören“ auf das wirkmächtige, weil von schöpferischer und heilswirksamer Tat untermauerte Schweigen des Bischofs (IgnEph 6,1), ja Gottes selbst (ebd. 15,1) aufgebaut.

Die spezifische Lehre, gegen die „Ignatios“ in IgnTrall polemisiert, ist eine Art Doketismus, die behauptet, Jesus Christus habe nur scheinbar körperlich gelebt, gelitten und den Tod erlitten (IgnTrall 9f.). Wer so argumentiere, so „Ignatios“, vertrete letztlich einen Glauben ohne Christus. Er verweist deshalb (IgnTrall 10) auf

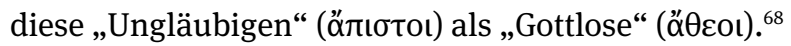

Er beschließt den Brief mit einem erneuten Plädoyer an seine Adressaten um

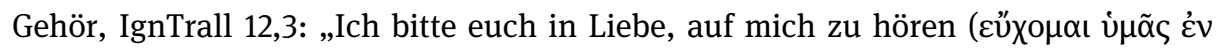

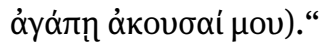

In IgnRom wird, wie bereits gesehen - ähnlich wie in IgnEph - die Thematik sehr stark entwickelt. Bereits IgnRom 2,1 findet sich der interessante Vergleich:

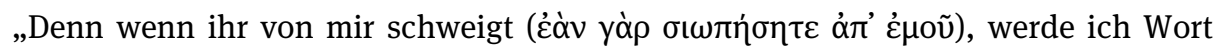
Gottes ( $\lambda$ óyos $\theta \varepsilon$ oṽ) sein; wenn ihr aber mein Fleisch liebt, werde ich wiederum ein Laut ( $\varphi \omega v \eta \dot{)}$ sein. “69

Das Wort „schweigen“ ( $\sigma \iota \omega \pi \tilde{\alpha} v)$ wird hier bereits zum zweiten Mal in rascher Abfolge verwendet. Im Satz vorher waren die Römer ermahnt worden, sie sollten darauf aus sein, Gott zu gefallen, nicht den Menschen. ${ }^{70}$ Wenn sie schwiegen (ćàv $\sigma \iota \omega \pi \eta \dot{\sigma} \tau \tau)$, würden sie sich dadurch in ein Unternehmen ("丷pyov) „einschreiben“

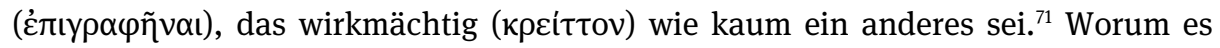

68 Zur Unbestimmtheit und möglichen Falschdarstellung des hier erwähnten „Doketismus“ s. die ausführliche Diskussion bei Schoedel (1990) 255-257.

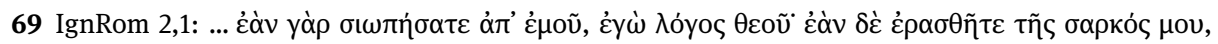
$\pi \alpha \dot{\lambda} \mathrm{\iota v} \varepsilon ̋ \sigma o \mu \alpha \iota \varphi \omega v \eta ́$.

70 Eine Anspielung auf 1Thess 2,4 und Gal 1,10; s. Schoedel (1990) $274 \mathrm{f}$.

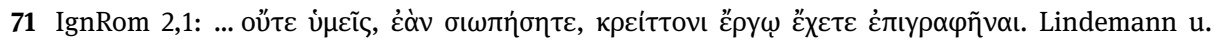

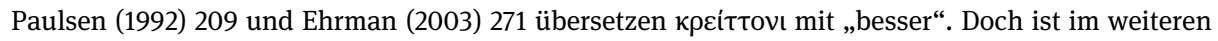
Kontext ja von der Wirkmächtigkeit des aus dem Schweigen geborenen Wortes Gottes die Rede, das von der Handlung des Märtyrers bezeugt wird, nicht in leerer Rede. Insofern sich „Ignatios”“ Adressaten diesem Unternehmen „verschreiben“, sind ihre Namen sozusagen wie in einen Gedenkstein

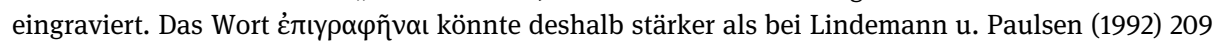


„Ignatios“ hier im Klartext geht, ist, dass er nicht will, dass sich die Römer für ihn einsetzen, um sein Martyrium zu verhindern. Sollten sie sein fleischliches Leben retten, würden sie ihn zu einem bloßen „Laut“ ( $\varphi \omega v \eta ́)$ reduzieren. Er könnte dann nicht das „Wort (入óyoৎ) Gottes“ sein, das er hofft durch seine Tat zu werden. Die Aussagekraft seines Martyriums wird demnach sozusagen durch das Schweigen seiner Adressaten wie auch durch sein eigenes Schweigen untermauert.

In IgnRom 3,2 wird dieser Gedanke weitergeführt, wenn „Ignatios“ bittet, die

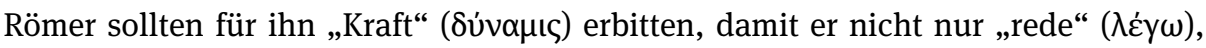
sondern auch „wolle“ ( $\theta \dot{\varepsilon} \lambda \omega)$, und nicht nur „Christ“ genannt ( $\lambda \dot{y} \gamma o \mu \alpha \mathrm{l})$, sondern

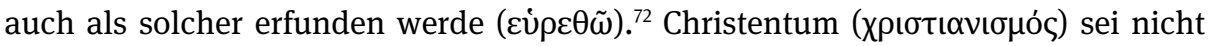
Sache von Überredung ( $\pi \varepsilon เ \sigma \mu o v \eta ́)$, sondern von „Größe“, oder vielleicht besser

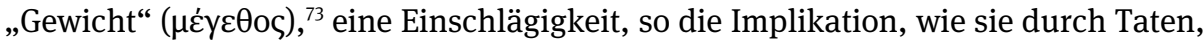
nicht durch bloße Worte erzielt wird..$^{74}$

Doch beinahe wie um sich selbst zu widersprechen, entwickelt „Ignatios“ in den nun folgenden Paragraphen eine geradezu überzogene Rhetorik, mit der er seine Adressaten dazu überreden will, ihn nicht vom Martyrium abzuhalten. In

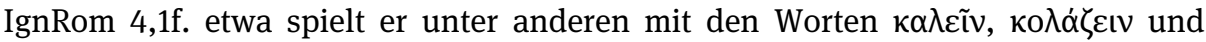

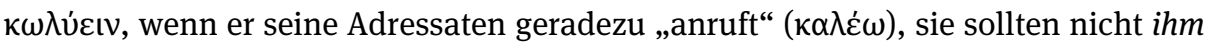

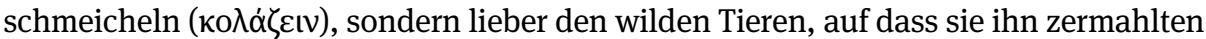

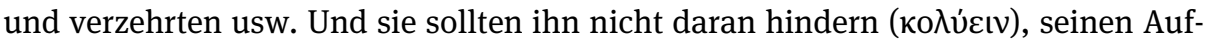

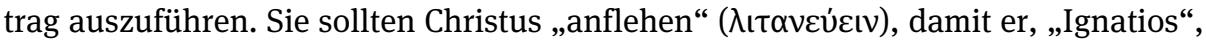

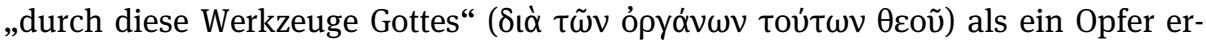

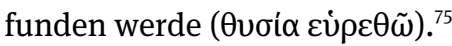

(„beisteuern“) übersetzt werden; s. etwa Ehrman (2003) 271: „enlisted“; zur Bedeutung dieser Art „epigraphischen“ Denkens im frühen Christentum s. Lössl (2014).

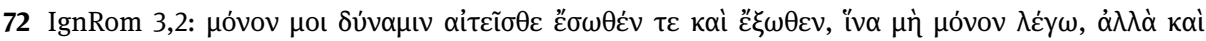

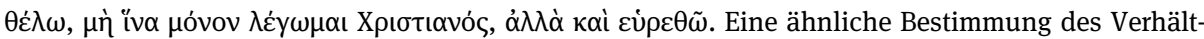

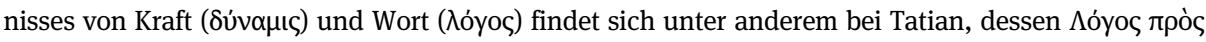
"E $\lambda \lambda \eta v \alpha \varsigma$ in etwa zeitgleich zu den Ignatianen entstand; s. dazu Lössl (2010).

73 IgnRom 3,3. Der Kontext würde erneut nahelegen, dass mit $\mu \varepsilon \dot{y \varepsilon} \theta$ o nicht so sehr „Größe“ im Sinne von ethischer Erhabenheit gemeint sein dürfte, wie die Übersetzungen von Lindemann u. Paulsen (1992) 211 und Ehrman (2003) 273 suggerieren, sondern rhetorische Wirkung und Einschlagskraft.

74 IgnRom 3,3. Der Gedankengang ist hier erneut ausführlicher und komplexer, als er im begrenzten Rahmen dieses Aufsatzes dargestellt werden kann: Das Christentum ist von Gewicht, so „Ignatios“,

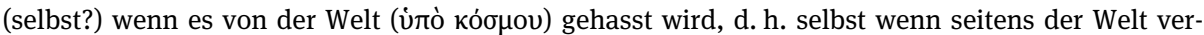
sucht wird, es niederzuhalten und auszumerzen; denn, so impliziert „Ignatios“, es gehe hier nicht um etwas oberflächlich Sichtbares, Scheinbares, sondern um die Manifestation von etwas in seinem Wesen Verborgenem: „Nichts Sichtbares“, so schreibt er, „ist gut. Denn unser Gott, Jesus Christus,

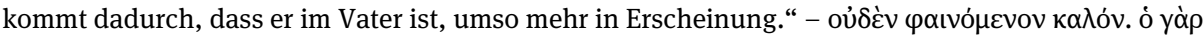

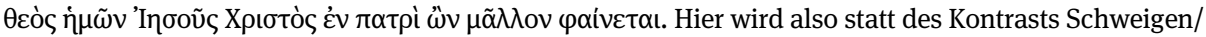
Reden der Kontrast Verborgenheit/Offenbarung bemüht. Der Effekt ist jedoch derselbe und die

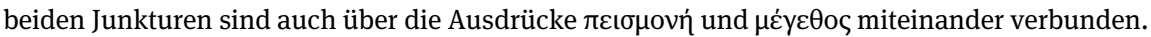

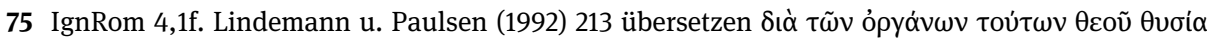
$\varepsilon \dot{v} \rho \varepsilon \theta \tilde{\omega}$ mit „durch diese Werkzeuge als Opfer Gottes“, während Ehrman (2003) 275 den Genitiv 
Es folgt der oben bereits besprochene Abschnitt IgnRom 4,3, wo „Ignatios“ von sich sagt, dass er seinen Adressaten nicht wie Petrus und Paulus Befehle erteile

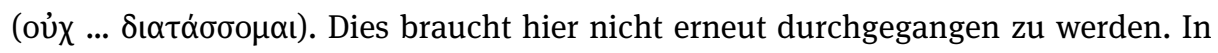
IgnRom 5 und 6 treten die Motive Reden, Schweigen und Hören in den Hintergrund. IgnRom 5 ergeht sich in geradezu parodistischer Weise in der Vorstellung, dass

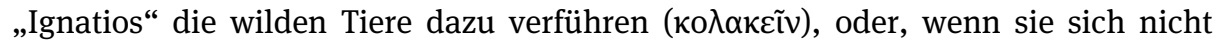

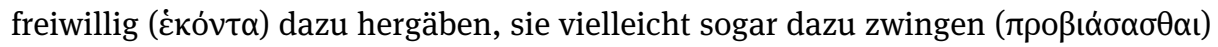

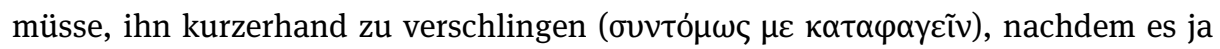
doch auch schon vorgekommen sei, dass einige ihre Opfer aus Feigheit nicht angerührt hätten.$^{76}$ Es folgt in IgnRom 6 eine vertiefte theologische Deutung des antizipierten Martyriums, wobei sowohl in IgnRom 5 als auch in IgnRom 6 paulinische Motive etwa aus 1Kor und Kol herangezogen werden. ${ }^{77}$ In IgnRom 7 drängen dann die Motive Reden, Schweigen und Hören wieder stärker in den Vordergrund.

IgnRom 7,1 endet mit der Aufforderung: „Sagt nicht ,Jesus Christus‘, während

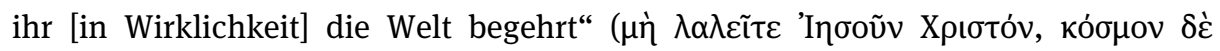
غ̇лı $\theta \mu \varepsilon \tilde{\tau} \tau \varepsilon)$. Hier ist wieder der schon vertraute Gegensatz zwischen „Rede“ und Wirklichkeit. Und es folgt ein angesichts des pseudepigraphischen Charakters des Corpus äusserst aufschlussreicher Satz. „Ignatios“ fordert seine Adressaten auf:

Lasst euch nicht von mir als jemand überzeugen, der persönlich gegenwärtig ist, wenn ich euch auffordere; sondern lasst euch vielmehr von dem überzeugen, was ich euch schreibe. $-\mu \eta \delta^{\prime}$ àv

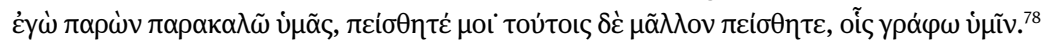

Der Verfasser dieser Zeilen empfiehlt sich als jemand, der nicht von dem von ihm produzierten Text verschieden, sondern mit ihm identisch ist. Ignatios, das ist nicht jemand, irgendeine Person, die sich auf dem Weg nach Rom befindet, um dort von

plausibler auf die Werkzeuge bezieht. $\varepsilon \dot{v} \rho \varepsilon \theta \tilde{\omega}$ findet sich an dieser Stelle bereits zum vierten Mal

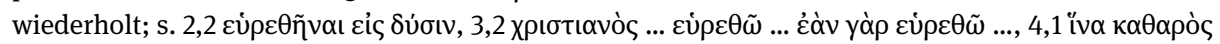

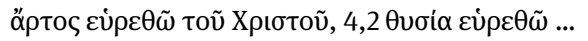

76 IgnRom 5,2. Dass Tiere in der Arena ihre intendierten Opfer nicht immer mit der von ihnen erwarteten Aggressivität angriffen, sei es dass sie durch den dort herrschenden Lärm desorientiert oder sonst irgendwie abgelenkt wurden, scheint einerseits historisch belegt zu sein, andererseits ist es offenbar ein Topos der frühchristlichen Martyriumsliteratur; vgl. etwa Passio Perpetuae 19,6; Acta Pauli et Theclae 32-35; Eus., hist. eccl. 5,1,42, zitiert bei Schoedel (1990) 288 mit weiteren Diskussionspunkten.

77 S. etwa IgnRom 5,1 „Ignatios'“ Aussage, er sei trotz der von ihm erlittenen Misshandlungen, die

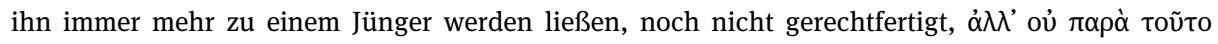

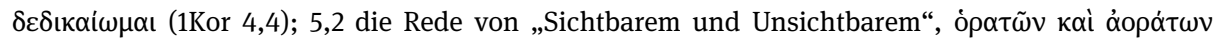
(Kol 1,16); 6,1 „Ignatios““ Aussage, „,schön [passend, angemessen] ist es für mich, zu sterben“, к $\alpha \lambda$ òv

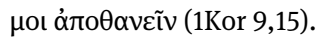

78 Natürlich handelt es sich hier um die Variation eines verbreiteten Brieftopos, nämlich der Brief sozusagen als Ersatz für die Anwesenheit des Korrespondenten; s. dazu Bauer (2011) 39f. Dennoch ist es eigenartig, wie ausführlich und dezidiert „Ignatios“ dieses Motiv an dieser Stelle entwickelt. 
Tieren aufgefressen zu werden und so das Martyrium zu erleiden; oder gar jemand, der zum Zeitpunkt, da der Brief gelesen wird, das Martyrium bereits erlitten hat und schon längst tot ist. Ignatios, das ist vielmehr dieser Text, dieser Brief, dieses BriefCorpus, durch dessen Rezeption in den Adressaten jene Liebe erweckt wird, die ein solches Zeugnis, wie es in diesem Corpus propagiert wird, überhaupt erst ermöglicht. Nicht um die Historizität des Martyriums des Ignatios also geht es in dem Corpus. Gerade das betont der Verfasser ja immer wieder: Er ist eben (noch) kein Märtyrer, sondern nur jemand, der ein solcher werden will. „Denn als Lebender schreibe

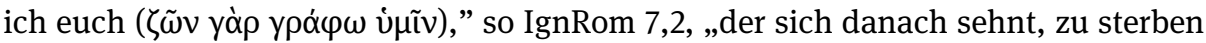

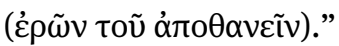

Also nicht mit Hagiographie oder Martyrologie haben wir es hier eigentlich zu tun. Die Briefe scheinen nicht in erster Linie in der Absicht abgefasst zu sein, den Adressaten von den Taten des Ignatios zu berichten, die ja zum Zeitpunkt, da die Briefe geschrieben sein wollen, noch ausstehen, wie der Verfasser selbst immer wieder betont; und vielleicht ist dies ja auch der Grund, warum das antizipierte Martyrium etwa in IgnRom 5 auf solch übertriebene und geradezu parodistische Weise beschrieben wird. Worum es in den Ignatianen, wie es scheint, in erster Linie geht, ist nicht die Martyriumsbegeisterung oder das Martyrium des Ignatios, sondern die Martyriumsbegeisterung seiner Adressaten, die geweckt werden soll, „nicht

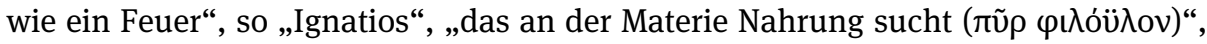
sondern „wie ein lebendiges Wasser (

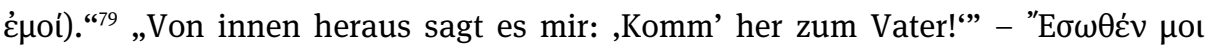

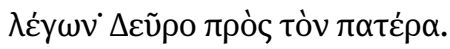

Es spricht hier also nicht der Mensch Ignatios, sondern durch ihn, von jenseits, von innen her, wie aus der aus sich heraus aufwallenden Quelle, von der auch in Joh 4,10 und 7,38 die Rede ist, werden die Adressaten angesprochen. In IgnRom 8,1 werden sie aufgefordert, ihr Leben zu ändern. Nicht mehr nach Menschenart sollen sie leben. Die Fähigkeit dazu werde ihnen verliehen, solange sie nur ihren Willen dazu bereiteten: „Dies aber wird geschehen, wenn ihr es wollt. Wollt, auf dass auch

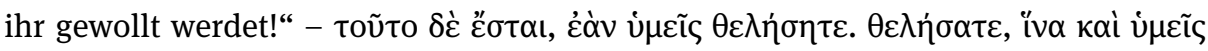
$\theta \varepsilon \lambda \eta \theta \tilde{\eta} \tau \varepsilon .{ }^{80}$ Dieses Wollen und gewollt Werden ist nicht blind. Es gründet auf Wahrheit: Wenn die Adressaten der Botschaft des an sie gerichteten Briefs, jener

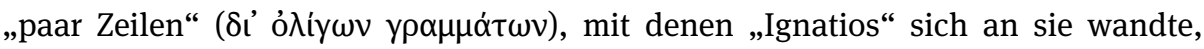

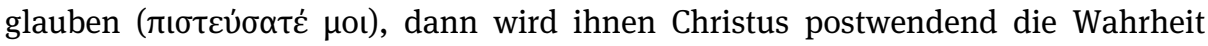
dessen offenbaren, was „Ignatios“ ihnen in IgnRom vermitteln wollte: 'Inбoũ $\delta \dot{\varepsilon}$

79 IgnRom 7,2. Zur Frage ob mit ṽ $\delta \omega \rho \zeta \tilde{\omega} v(J o h$ 4,10; 7,38) eine direkte Abhängigkeit von Joh vorliegt, s. Nagel (2000) 232-234.

80 IgnRom 8,1. 


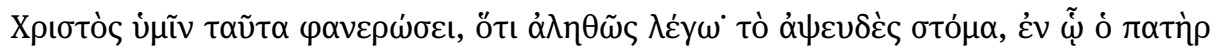
$\dot{\alpha} \lambda \eta \theta \tilde{\omega} \varsigma \dot{\varepsilon} \lambda \alpha \dot{\lambda} \eta \sigma \varepsilon v .^{81}$

Erneut wird hier also jener Zirkel sichtbar: Das Reden, Anreden und Zureden des Ignatios, das die Adressaten zum wahren Glauben an Christus bewegen will, der auch das Wollen impliziert, ein entsprechendes Leben (der Bereitschaft zum Martyrium) zu führen; und im Gegenzug eine Bewegung von Gott (vom Jenseits, vom Schweigen des Vaters) her, die die Wahrheit des Ganzen fundiert, aber nicht in jener Verborgenheit bleibt, sondern in Christus geoffenbart wird und dadurch die Rede des „Ignatios“ verifiziert.

In den verbleibenden Briefen, IgnPhld, IgnSm und IgnPol, kommen all diese Motive erneut weniger $\mathrm{zu}$ tragen als in IgnEph und IgnRom. Es sollen hier aber der Vollständigkeit halber dennoch einige Beispiele angeführt werden, auch um die Präsenz dieser Motive im ganzen Corpus zu illustrieren.

IgnPhld 1,1 etwa beginnt mit einem Lob des Bischofs, „der schweigend mehr zu-

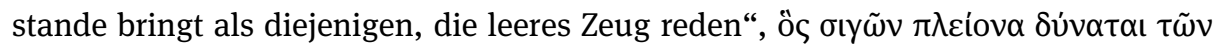

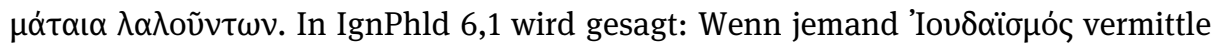

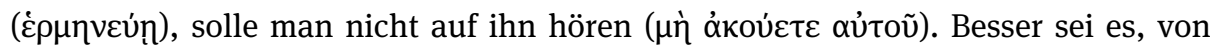

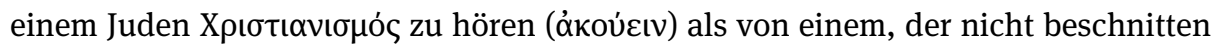

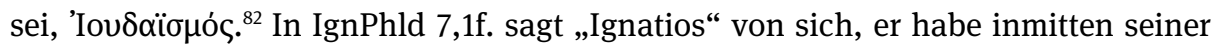

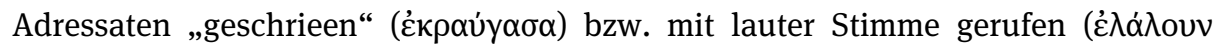
$\mu \varepsilon \gamma \alpha \dot{\lambda} \lambda \eta \emptyset \varphi v \tilde{n})$, d. h. mit Gottes Stimme ( $\theta \varepsilon$ ṽ $\varphi \omega v \tilde{n})$, sie sollten sich an den Bischof halten (d. h. keine Konventikel bilden, in denen sie ihre christliche Praxis getrennt vom Bischof vollzögen und dadurch eine Spaltung verursachten).$^{83}$

Die Verbindung an dieser Stelle von „Schreien“, „Reden mit lauter Stimme“ und „Gottes Stimme“ erfolgt nicht zufällig. „Lautes Sprechen und Schreien ist ... in den Ignatianen Zeichen prophetischer und pneumatischer Rede." ${ }^{84}$ Es erfolgt wohl in Anspielung auf Lk 1,41f., den Ausruf Elisabeths bei der Begegnung mit Maria. Gleichzeitig erinnert die Rede von der Stimme Gottes an das Schweigen Gottes (бıyń,

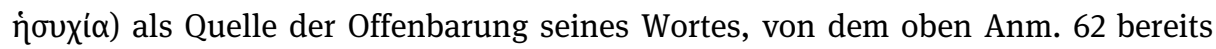
einmal die Rede war, im Zusammenhang mit IgnEph 15,1f.

In IgnPhld 8,2 erfolgt dann noch einmal ein mahnender Aufruf ( $\pi \alpha \rho \alpha \kappa \alpha \lambda \tilde{\omega} \delta \dot{\varepsilon}$ v่ $\tilde{\alpha} \varsigma$ ) im Kontext einer Polemik gegen Quellenkritiker, die nicht glauben wollen, was sie nicht in den Urkunden finden können. ${ }^{85}$ Erneut betont „Ignatios“ hier das

81 IgnRom 8,2.

82 Zur Signifikanz der ,anti-judaisierenden“ Stellen in den Ignatianen s. unten Anm. 85.

83 Zur Bedeutung des Schreiens an dieser Stelle s. Lechner (1999) 257-261.

84 Lechner (1999) 259.

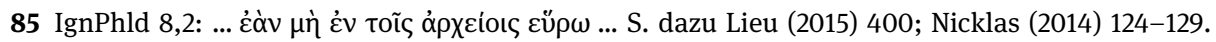
Theobald (2016) 313 interpretiert sämtliche „anti-judaisierenden“ Stellen in IgnMagn 8,1-10,3 und Phld 6,1f.; 8,2 unter der Rücksicht des „Rom-Bezugs“ der Ignatianen. Das Plädoyer für den Herrentag in IgnMagn sei eine Stellungnahme bezüglich des Ostertermins. Die Berufung auf Polykarp sei 
Leben, Leiden und den Kreuzestod Jesu Christi sowie seine Auferstehung und den Glauben an ihn als Wahrheitskriterien für seine Botschaft, nicht bestimmte Texte oder Urkunden.

In IgnSm 5 wird das Leugnen dieser Glaubensinhalte als negative Haltung mit dem Tod verglichen. Diese Leugner, so „Ignatios“, seien „Wortführer des Todes“

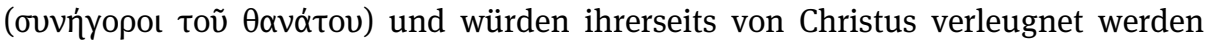

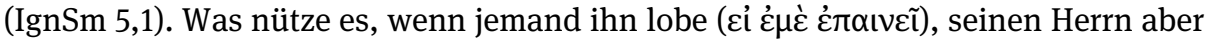

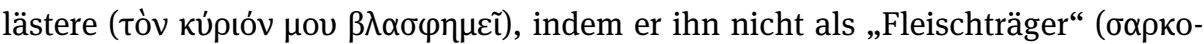

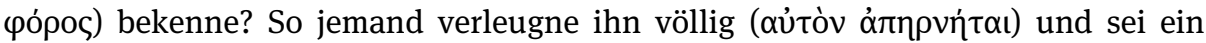

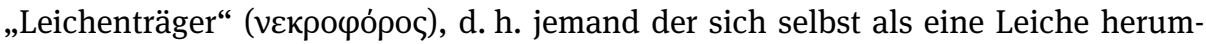
schleppe $(5,2)$.

In IgnPol 3,1 wird diese Topik aufgegriffen und zur Gegenwehr aufgerufen: „Die vertrauenswürdig zu sein scheinen und doch Irrtümer verbreiten, sie sollen dich

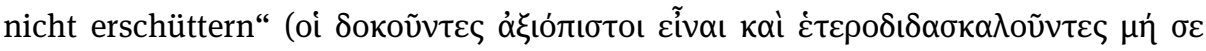
$\kappa \alpha \tau \alpha \pi \lambda \eta \sigma \sigma \varepsilon ́ \tau \omega \sigma \alpha \nu)$, rät „Ignatios“ Polykarp; und es folgen Ermahnungen zu vermehrtem Eifer bzw., besser, zu größerer Sorgfalt und Gewissenhaftigkeit beim Inter-

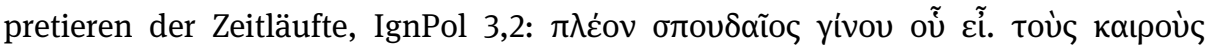
$\kappa \alpha \tau \alpha \mu \alpha \dot{v} \theta \alpha \nu \varepsilon$. Als Kriterium für eine scharfsinnigere und zutreffendere Interpretation der Zeitereignisse nennt „Ignatios“, wie er es bereits an früheren Stellen getan hat, ${ }^{86}$ den überzeitlichen, unsichtbaren, leidensunfähigen Gott, der um unseretwillen zeitlich, sichtbar und leidensfähig wurde und tatsächlich auch gelitten hat. ${ }^{87}$ Auf dieser Grundlage, so impliziert „Ignatios“ hier, kann Polykarp nicht nur die „schlim-

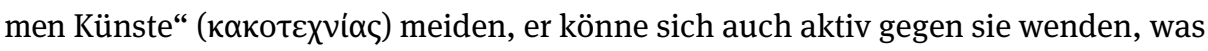

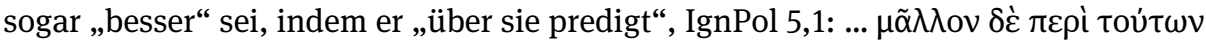

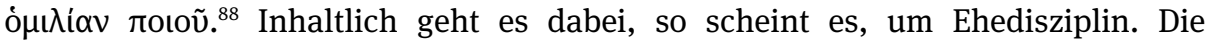

„pikant“: „Der ... vielleicht römische Autor instrumentalisiert mit seiner Fälschung die Autorität der Bischöfe von Antiochia und Smyrna ... für die Anliegen Roms und versucht, dort ... das durchzusetzen, was im Osten schon gute Praxis sei: den Monepiskopat.“ Dies könnte auch Irenäus' Sympathie für das Werk und seinen Autor erklären; s. unten Anm. 93f.

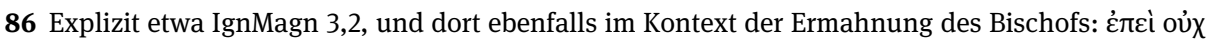

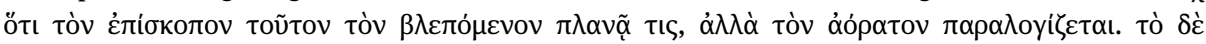

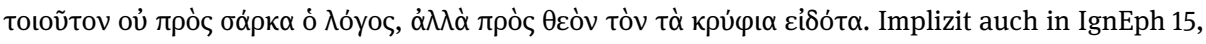
wo der verborgene Gott als Quelle wahrer Erkenntnis beschrieben wird.

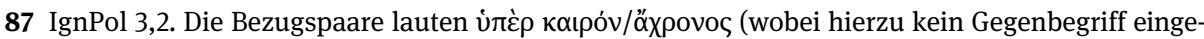

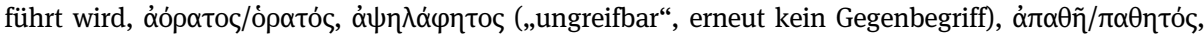

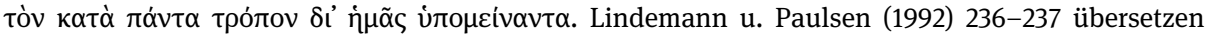

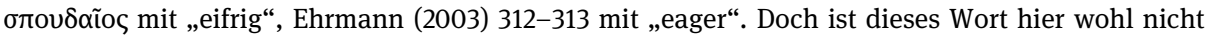
nur ethisch, sondern auch erkenntnistheoretisch zu verstehen, im Sinne etwa von „scharfsinniger“, „aufmerksamer“ sein (im Interpretieren der Zeitereignisse).

88 Es ist dies eine der frühesten Stellen in der frühchristlichen Literatur, wo ò $\mu \lambda \lambda \dot{\alpha} \alpha$ im Sinne von „Predigt“ gebraucht wird. Zur ursprünglichen Verwendung des Wortes in dieser Bedeutung im Kontext der Schulphilosophie s. oben Anm. 59. 
„Schwestern“ sollen sich an ihren Lebensgefährten genügen lassen und umgekehrt. Was hier erneut interessiert, sind die Vokabeln des Redens und Zuredens, die „Ignatios“ verwendet, um zu beschreiben, wie sich Polykarp an seine Adressaten wenden soll, wenn er predigt: Mit den Schwestern solle er reden $(\pi \rho 0 \sigma \lambda \alpha \lambda \varepsilon \tilde{v}),{ }^{89}$ die Brüder solle er auffordern ( $\pi \alpha \rho \alpha y y \varepsilon ́ \lambda \lambda \varepsilon เ v)$ mit dem Ziel, dass sich niemand seiner

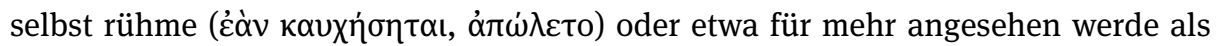

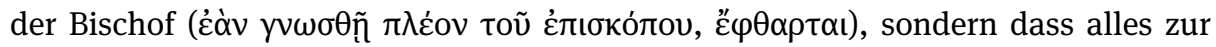

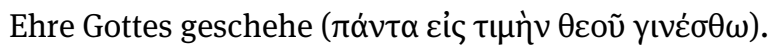

Die homiletische Kommunikation zwischen Polykarp als Bischof und seiner Gemeinde wird hier nach dem Modell der brieflichen Kommunikation zwischen „Ignatios“ und seinen Adressaten konzipiert. Dies wird am Ende des Briefes, in IgnPol 8,1, noch unterstrichen, wenn „Ignatios“ seinem Adressaten mitteilt:

Da ich nun nicht selbst an alle Kirchen schreiben konnte ..., sollst du ... den Kirchen schreiben, die noch vor mir liegen, weil du Gottes Sinn $(\gamma v \omega \dot{\mu} \mu \eta)^{90}$ besitzst, damit auch sie desgleichen tun:

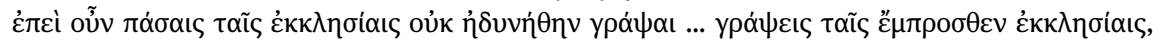

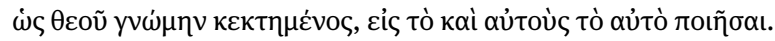

Mit diesem programmatischen Satz schließt sich der Kreis. „Ignatios“, d. h. der pseudepigraphische Verfasser des Siebener-Corpus, schrieb den Kirchen, um sich

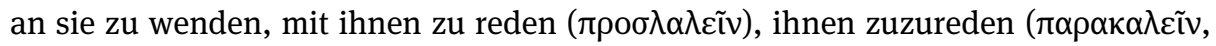
$\pi \alpha \rho \alpha ı \varepsilon \tau v)$, sie vielleicht auch aufzufordern ( $\pi \alpha \rho \alpha y \gamma \varepsilon \dot{\varepsilon} \lambda \varepsilon \sigma \theta \alpha \mathrm{s})$, in einigen Punkten vielleicht auch ihnen gegenüber $\mathrm{zu}$ insistieren ('́) $\left.\mathcal{c}^{\prime} \lambda \lambda \varepsilon \sigma \theta \alpha \mathrm{l}\right)$, generell aber, um ihnen Dinge ans Herz zu legen, die er nur dann, wenn er ein Apostel wie Petrus und Paulus gewesen wäre, auch hätte „befehlen“ ( $\delta \iota \alpha \tau \alpha ́ \sigma \sigma \varepsilon \sigma \theta \alpha \iota)$ können, Dinge die die wahre Lehre ( $\gamma v \omega \dot{\mu} \mu)$ betrafen sowie die Zurückweisung falscher Lehren (wie Gnosis, Doketismus, judaisierende Tendenzen), die Autorität des Bischofs und die tiefere Bedeutung der Blutzeugenschaft - insbesondere im Hinblick auf das Geheimnis des Leidens und Sterbens Christi, das die Adressaten angehalten werden zu verinnerlichen, gemäß dem Vorbild des Absenders.

Wenn er in IgnPol Polykarp persönlich dazu auffordert, ihn in dieser Hinsicht nachzuahmen, konstruiert er Polykarp sozusagen als Scharnier, das seine Briefe mit der Realgeschichte verbindet. Nach Theobald war es zuallererst der Brief des Polykarp nach Philippi, der „dem anonymen Autor der Ignatianen den Anstoß zu seiner raffinierten Fälschung“ gab. Die Erwähnung des Martyriums des Ignatios in Polyc 9,1f. „brachte ihn auf die Idee“, dessen Figur „zu einem literarischen Bild des vor-

89 Zur Bedeutung von $\pi \rho \circ \sigma \lambda \alpha \lambda \varepsilon \tilde{v}$ im Unterschied zu $\pi \alpha \rho \alpha \kappa \alpha \lambda \varepsilon \tilde{v}$ in diesem Zusammenhang s. bereits oben Anm. 49.

90 Zur Bedeutung von yvú ${ }$, das Lindemann u. Paulsen (1992) 241 mit „Sinn“ übersetzen, s. oben Anm. 51, 63 und 64. 
bildlichen Märtyrerbischofs auszubauen“91, eines Märtyrerbischofs aus dem syrischen Osten, der, wie es scheint, im Osterstreit eine römische Position vertritt und für Rom den Monepiskopat propagiert. ${ }^{92}$

Es war das Anliegen der vorliegenden Untersuchung, anhand der angeführten Beispiele aufzuzeigen, wie er etwa durch die Anwendung der erwähnten Begriffe in den gegebenen Kontexten nach den Regeln antiker Briefkommunikation dabei vorging. Die zitierten Beispiele dürften ihn als einen im Großen und Ganzen recht kompetenten Briefkommunikator erwiesen haben, der nicht nur ein literarisches Produkt von hoher Qualität produzierte, sondern auch mehr oder weniger direkt mögliche Adressaten ansprach. Die früheste Rezeption, etwa bei Irenäus (adv. haer. 5,28,4), die eine positive Reaktion darstellt, scheint dies zu bestätigen. Irenäus vermeidet es, Ignatios als den Verfasser des Werks zu nennen, aus dem er zitiert. Er nennt ihn

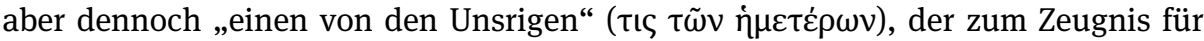

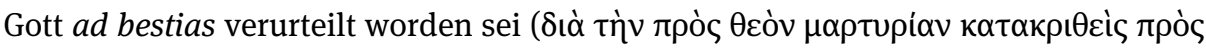
$\theta \eta \rho i \alpha)$ und sein Martyrium mit diesen Worten gedeutet habe: „Ich bin Weizen Gottes und werde von den Zähnen der wilden Tiere zermahlen, damit ich als reines Brot erfunden werde." $" 93$

Irenäus eignet sich hier also nicht nur ein vereinzeltes Theologoumenon aus den Ignatianen an, er akzeptiert nebenbei auch dessen Verfasser als „einen der Unsrigen“. Damit muss er in ihm nicht notwendigerweise einen „Parteigänger“ oder Landsmann (aus Kleinasien) gesehen haben, wie Theobald vermutet, ${ }^{94}$ der Ausdruck könnte auch einfach umschreiben, dass es sich bei dem Verfasser um einen Mitchristen handelte. Dennoch könnte die Tatsache, dass Irenäus ihn nicht beim Namen nennt, ein Indiz dafür sein, dass er sehr wohl weiss, oder zumindest ahnt, dass die Briefe pseudepigraphisch sind. ${ }^{95}$

Die vorliegende Untersuchung kann dem nicht weiter nachgehen. Es soll nur noch einmal wiederholt werden, dass diese reale Möglichkeit der Wirkung der Ignatianen als Brief-Corpus (im theologischen wie im literarischen Sinne), von der frühesten Rezeption angefangen, keinerlei Abbruch getan hat.

91 Theobald (2016) 323.

92 Theobald (2016) 313.

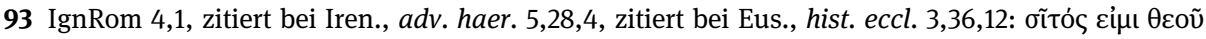

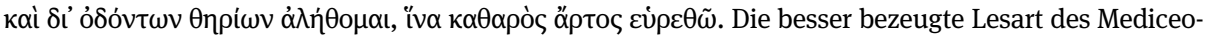

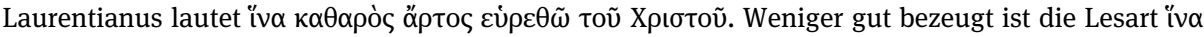

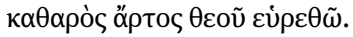

94 Theobald (2016) 312 Anm. 225.

95 Theobald (2016) 312 Anm. 225 spricht davon, „dass er der Figur des Ignatios gegenüber skeptisch war“, was Ignatios' Verfasserschaft des Corpus betrifft. 


\section{Literaturverzeichnis}

Ayán Calvo (2002): Juan José Ayán Calvo, „Datierung und Echtheit der Ignatianischen Briefe“, FKTh 18, 81-105.

Barnes (2008): Timothy D. Barnes, „The Date of Ignatius“, ET120, 119-130.

Barnes (2010): Timothy D. Barnes, Early Christian Hagiography and Roman History, Tübingen.

Bauer (2011): Thomas Johann Bauer, Paulus und die kaiserzeitliche Epistolographie. WUNT 276, Tübingen.

Bihlmeyer (1956): Karl Bihlmeyer (Hg.), Die Apostolischen Väter, zweite Auflage, erster Teil, Tübingen. Bonhöffer (1911): Adolf Bonhöffer, Epiktet und das Neue Testament. RVV 10, Gießen.

Brent (2006): Allen Brent, Ignatius of Antioch and the Second Sophistic. A Study of an Early Christian Transformation of Pagan Culture. STAC 36, Tübingen.

Brent (2009): Allen Brent, Ignatius of Antioch: A Martyr Bishop and the Origin of Episcopacy, London.

Brox (1976): Norbert Brox, „Pseudo-Paulus und Pseudo-Ignatius. Einige Topoi altchristlicher Pseudepigraphie“, VigChr 30, 181-188.

Bultmann (1967): Rudolf Bultmann, „Ignatius und Paulus“, Ders., Exegetica: Aufsätze zur Erforschung des Neuen Testaments, hg. von Erich Dinkler, Tübingen, 400-411.

Burke (2013): Tony Burke (Hg.), Ancient Gospel or Modern Forgery? The Secret Gospel of Mark in Debate, Eugene, Oregon.

Dallaeus (1666): Ioannes Dallaeus, De scriptis quae sub Dionysii Areopagitae et Ignatii Antiochenis nominibus circumferuntur libri duo, Genf.

Dehandschutter (2009): Boudewijn Dehandschutter, „Der Polykarpbrief“, Wilhelm Pratscher (Hg.), Die Apostolischen Väter. Eine Einleitung, Göttingen, 130-146.

Ehrman (2003): Bart D. Ehrman, The Apostolic Fathers, Vol. 1. LCL 24, Cambridge, MA u. London.

Flacius (1559): Matthias Flacius u. a., Ecclesiastica Historia. Cent. 1-3, Basel.

Glucker (1978): John Glucker, Antiochus and the Late Academy. Hyp. 56, Göttingen.

Gryson (1979): Roger Gryson, „Les ,lettres' attribuées à Ignace d'Antioche et l'apparition de l'épiscopat monarchique“, RTL 10, 446-453.

Hübner (1997): Reinhard Hübner, „Thesen zur Echtheit und Datierung der sieben Briefe des Ignatius von Antiochien“, ZAC 1, 44-72.

Hübner (1999): Reinhard Hübner, Der paradox Eine: Antignostischer Monarchianismus im zweiten Jahrhundert. SVigChr 50, Leiden.

Huller u. Gullotta (2017): Stephan Huller u. Daniel Nicholas Gullotta, „Quentin Quesnell's Secret Mark Secret“, VigChr 71, 252-378.

Joly (1979): Robert Joly, Le dossier d'Ignace d'Antioche, Brüssel.

Killen (1886): William P. Killen, The Ignatian Epistles Entirely Spurious. A Reply to the Rt. Rev. Dr. Lightfoot, Bishop of Durham, Edinburgh.

Lagarde (1882): Paul de Lagarde, Die lateinischen übersezungen [sic!] des Ignatius. AGWG 29, Göttingen.

Lampe (2003): Peter Lampe, From Paul to Valentinus. Christians at Rome in the First Two Centuries, London.

Lechner (1999): Thomas Lechner, Ignatius adversus Valentinianos? Chronologische und theologiegeschichtliche Studien zu den Briefen des Ignatius von Antiochien. SVigChr 47, Leiden.

Lieu (2015): Judith Lieu, Marcion and the Making of a Heretic. God and Scripture in the Second Century, Cambridge.

Lightfoot ('1889): Joseph B. Lightfoot, The Apostolic Fathers, Pt. II: S. Ignatius, S. Polycarp, vol. 1, 2nd ed., London. 
Lindemann u. Paulsen (1992): Andreas Lindemann u. Henning Paulsen (Hgg.), Die apostolischen Väter. Griechisch-deutsche Parallelausgabe, auf der Grundlage der Ausg. von Franz Xaver Funk, Karl Bihlmeyer und Molly Whittaker. Mit Übers. von M. Dibelius und D.-A. Koch neu übers. u. hg. von Andreas Lindemann u. Henning Paulsen, Tübingen.

Löhr (2009): Hermut Löhr, „Die Briefe des Ignatius von Antiochien“, Wilhelm Pratscher (Hg.), Die Apostolischen Väter. Eine Einleitung, Göttingen, 104-129.

Lössl (2010): Josef Lössl, „Zwischen Christologie und Rhetorik. Zum Ausdruck ,Kraft des Wortes“ ( (Hgg.), Logos der Vernunft - Logos des Glaubens. MS 31, Berlin u. New York, 129-147.

Lössl (2014): Josef Lössl, „An Inextinguishable Memory: ,Pagan“ Past and Presence in Early Christian Writing“, Carol Harrison u. a (Hgg.), Being Christian in Late Antiquity (FS Gillian Clark), Oxford, 74-89.

Merz (2004): Annette Merz, Die fiktive Selbstauslegung des Paulus. Intertextuelle Studien zur Intention und Rezeption der Pastoralbriefe. NTOA/StUNT 52, Göttingen

Mitchell (2006): Matthew W. Mitchell, „In the Footsteps of Paul: Steps Along the Road to Canon in Ignatius of Antioch", JECS 14, 27-46

Nagel (2000): Titus Nagel, Die Rezeption des Johannesevangeliums im 2. Jahrhundert. Studien zur vorirenäischen Aneignung und Auslegung des vierten Evangeliums in christlicher und christlichgnostischer Literatur, Leipzig.

Nicklas (2014): Tobias Nicklas, Jews and Christians? Second Century, Christian“ Perspectives on the ,Parting of the Ways', Tübingen.

Parvus (2008): Roger Parvus, A New Look at the Letters of Ignatius of Antioch and other Apellean Writings, New York.

Prostmeier (2002): Ferdinand Prostmeier, Art. „Ignatius von Antiochien“, $L A C L^{3} 346-348$.

Rathke (1967): Heinrich Rathke, Ignatius von Antiochien und die Paulusbriefe. TU 99, Berlin.

Rius-Camps (1995): Josep Rius-Camps, „Indicios de una redacción muy temprana de las cartas auténticas de Ignacio (ca. 70-90 d. C.)“, Aug. 34, 199-214

Ruinart (1689): Thierry Ruinart, Acta primorum martyrum sincera et selecta, Lyon.

Schmithals (2009): Walter Schmithals, „Zu Ignatius von Antiochien“, ZAC 13, 181-203.

Schoedel (1990): William R. Schoedel, Die Briefe des Ignatius von Antiochien. Ein Kommentar, München (aus dem amerikanischen Englisch übersetzt von G. Koester).

Sieben (1978): Hermann Josef Sieben, „Die Ignatianen als Briefe. Einige formkritische Bemerkungen“, VigChr 32, 1-18.

Theobald (2016): Michael Theobald, Israel-Vergessenheit in den Pastoralbriefen. Ein neuer Vorschlag zu ihrer historisch-theologischen Verortung im 2. Jh. $n$. Chr. unter besonderer Berücksichtigung der Ignatius-Briefe, Stuttgart.

Trevett (1983): Christine Trevett, „Prophecy and Anti-Episcopal Activity: A Third Error Combatted by Ignatius“, JEH 34, 1-18.

Voss (1646): Isaacus Vossius, Epistolae genuinae S. Ignatii martyris quae nunc primum lucem vident ex bibliotheca Florentina, Amsterdam.

Zahn (1873): Theodor Zahn, Ignatius von Antiochien, Gotha.

Zwierlein (2009): Otto Zwierlein, Petrus in Rom. Die literarischen Zeugnisse. UALG 96, Berlin u. New York. 